\title{
Multi-omics Analyses Revealed Key Factors Involved in Fluorescent Carbon-Dots-Regulated Secondary Metabolism in Tetrastigma Hemsleyanum
}

\section{Xin Peng}

Ningbo research institute of Zhejiang University

\section{Zhuomi Xie}

Fujian Agriculture and Forestry University

\section{Xiuhua Wang}

Ningbo Institute of Materials Technology and Engineering Chinese Academy of Sciences: Ningbo Institute of Industrial Technology Chinese Academy of Sciences

\section{Yuxiang Zhao}

Ningbo Research Institute of Zhejiang University

\section{Chuyun Yang}

Fujian Agriculture and Forestry University

\section{Zhongyi Zhang}

Fujian Agriculture and Forestry University

\section{Mingjie Li}

Fujian Agriculture and Forestry University

\section{Jianping Zheng}

Ningbo Institute of Materials Technology and Engineering Chinese Academy of Sciences: Ningbo Institute of Industrial Technology Chinese Academy of Sciences

Yuhui Wang ( $\sim$ wangyuhui@nimte.ac.cn )

Ningbo Institute of Materials Technology \& Engineering

\section{Research}

Keywords: Fluorescent carbon-dots, Tetrastigma hemsleyanum, Quality ingredients, Hub genes

Posted Date: November 23rd, 2021

DOI: https://doi.org/10.21203/rs.3.rs-1084632/v1

License: (c) (i) This work is licensed under a Creative Commons Attribution 4.0 International License. Read Full License

Version of Record: A version of this preprint was published at Journal of Nanobiotechnology on February 2nd, 2022. See the published version at https://doi.org/10.1186/s12951-022-01271-6. 


\section{Abstract}

Background: Luminescent nanomaterials (LNMs), especially newly-exploited fluorescent carbon-dots (CDs), have demonstrated promising candidates for sunlight harvesting and enhanced photosynthesis efficiency of crops. However, most of the studies focus on the design and synthesis of LNMs and primary metabolism in biomass acceleration, secondary metabolism that closely associated with the quality ingredients of plants is rarely mentioned.

Results: UV-absorptive and water-soluble NIR-CDs were harvested via a facile microwave-assisted carbonization method. The effect and regulatory mechanism of NIR-CDs on the secondary metabolism and bioactive ingredients accumulation in Tetrastigma hemsleyanum were explored. A total of 191 differential secondary metabolites and 6874 differentially expressed genes were identified when the NIR-CDs were adopted for enhancing growth of $T$. hemsleyanum. The phenolic acids were generally improved, but the flavonoids were more likely to decrease. The pivotal differentially expressed genes were involved in biosynthesis of secondary metabolites, flavonoid biosynthesis, porphyrin and chlorophyll metabolism, etc. The gene-metabolite association was constructed and 44 hub genes highly related to quality ingredients accumulation and growth were identified, among which and the top 5 genes of the PPI network might be the key regulators.

Conclusion: This research provided key regulatory genes and differentially accumulating quality ingredients under NIRCDs-treatment, which could provide a theoretical basis for expanding the applications of nanomaterial in industrial crop agriculture.

\section{Background}

At present, the whole world has reached a consensus that an effective and sustainable agricultural system is essential to the existence and development of humanity. Aiming to sustainably meet future demands for food, new agri-tech revolutions including information technology, new materials, and methodologies have largely emerged [1-4]. Among them, engineered nanomaterials (ENMs)-based nanotechnology positively leads this variation, which enhances the efficiency of cropping systems associated with all inputs via more efficient delivery mechanisms, and thereby improves the efficiency of nutrient utilization, and better management of disease and crop loss [5].

Photosynthesis is the most important energy conversion process on the earth due to its massive and green utilization of sunlight. ENMs reveals great potential to elevate plant photosynthesis efficiency through the following strategies i.e. avoiding abiotic stress [6], increasing chlorophyll content [7], enhancing sunlight harvesting and utilization [8], scavenging reactive oxygen species [9] and promoting electron transfer [10]. In virtue of photoluminescence character, luminescent nanomaterials (LNMs) are ideal candidates for the sunlight absorption and photon use [11]. Because of their small size and environmentally responsive release property, these LNMs possess the characteristics of good penetrability and diffusion in the plant vasculature after root or foliar applications [12]. Many types of LNMs, such as representative semiconductor-type quantum dots (QDs), fluorescent carbon dots (CDs), metal nanoclusters, lanthanide-doped up-conversion phosphors (UCPs), have been demonstrated great potentials in enhancing photon absorption in ultraviolet, red or near-infrared (NIR) region of the sunlight and elevated photosynthesis efficiency and crop yield [11]. Positive progresses have been achieved especially on the design or hybridization of these LNMs. For example, toxicity-reduced MPA-capped CdSe QDs, Si-QDs, PEI-coated UCPs, UCPs@CDs nanocomposites and diverse CDs (blue CDs-chloroplasts hybrid, bule/red dual-emissive CDs and NIR or far-red CDs) have been gradually developed and constructed for the enhanced sunlight absorption and photosynthesis efficiency [13-19]. Among the forementioned LNMs, as a newly-emerged zero-dimension nanophosphors, fluorescent CDs have attracted more attentions due to their fascinating merits e.g. facile and low-cost preparation, low toxicity, good physicochemical stability, excellent optical properties [20]. In our recently-reported work [21], we have produced the first comprehensive 
analysis of the light-harvesting effects and related mechanisms of NIR-CDs (as a preferential-selected LNMs) on primary metabolism of plant at gene level, which provides a method to research the mechanism of LNMs-caused increased sunlight absorption and photosynthesis efficiency. However, previous researches mainly focused on the design and fabrication of light-absorption nanomaterials, and the effect of these LNMs on growth of plant. There often exists an antagonistic effect between primary and secondary metabolism in plants, so more rapid photosynthesis may lead to the lower accumulation of nutritional or bioactive ingredients in plants. ENMs may be an effective approach to ensure vigorous primary metabolism that could remain a high level of biomass growth rate, but it is also essential and urgent to monitor the responses of secondary metabolism that would significantly influence the quality ingredients in plants $[5,11]$.

In recent years, technical advancements in metabolomics integrated with transcriptomics analyses have provided effective ways to explore the complex regulatory network in plants [22]. Weighted gene co-expression network analysis (WGCNA), a recently appeared approach in systems biology has been gradually applied to describe gene-related patterns under different treatment conditions, and to explore the key candidate genes involved in multiple metabolic pathways. Particularly, it has become an important tool in the identification of hub genes and the gene-metabolite association, then rebuild the gene regulatory sub-networks.

Herein, as a continued research work, the effect of NIR-CDs on the secondary metabolism and bioactive ingredients of Tetrastigma hemsleyanum (T. hemsleyanum, a class of medicinal plant) was determined, and the possible mechanism was also further explored by an integrated transcriptome and metabolome research. The gene-metabolite association were constructed by WGCNA, then the candidate hub genes highly related to quality ingredients accumulation and growth were identified. The results would lay the foundation for the application of nanotechnology in industrial crop agriculture.

\section{Results}

Characterizations of the NIR-CDs.

The NIR-CDs were prepared with a facile microwave-assisted carbonization method using GSH and formamide as the raw materials and solvent, respectively. To characterize the morphology, size, chemical composition and surface state of the harvested NIR-CDs, a series of measurements e.g. TEM, XRD, FT-IR, XPS, Raman and Zeta potential were severally performed. As seen from the TEM image (Fig. 1A), the NIR-CDs present very good mono-dispersity, uniform and spherical morphologies, and a narrow particle distribution with a mean size of $3.9 \mathrm{~nm}$ (Fig. 1B). Nonetheless, there are none lattice fringes are observed in the HR-TEM image (Fig. 1C), indicating that the NIR-CDs are mostly noncrystalline. In the XRD pattern (Additional files Fig. S1a), a typical peak at $26^{\circ}$ is assigned to the (002) plane of graphite, which further verifies the noncrystalline graphite structure of the NIR-CDs [23]. Furthermore, the graphite structure of NIR-CDs also confirmed by the Raman spectrum (Additional files Fig. S1b). As shown therein, two distinct peaks centered at 1550 and $1342 \mathrm{~cm}^{-1}$ represent the typical G-band and D-band, respectively. Simultaneously, a low ratio of $D$ to $G$ clearly authenticates the dominate pristine carbon in the NIR-CDs [24]. In the FT-IR spectrum of NIR-CDs (Fig. 1D), a broad and strong absorption band range from 3100 to $3500 \mathrm{~cm}^{-1}$ is attributed to the stretching vibrations of $\mathrm{O}-\mathrm{H}$ and $\mathrm{N}-\mathrm{H}$, implying the abundant existence of hydrophilic hydroxyl and amino groups. Typical stretching vibrations absorption peaks of $\mathrm{C}=\mathrm{O}$ and $\mathrm{C}=\mathrm{C} / \mathrm{C}=\mathrm{N}$ are easily observed at 1689 and $1583 \mathrm{~cm}^{-1}$, respectively. The peaks at 1160,1249 and $1390 \mathrm{~cm}^{-1}$ severally belong to the stretching vibrations of $\mathrm{C}-\mathrm{C}, \mathrm{C}-\mathrm{O}$ and $\mathrm{C}-\mathrm{N}$. The absorption band $\left(1000-1100 \mathrm{~cm}^{-1}\right)$ is assigned to $\mathrm{C}=\mathrm{S}$ and oxidized $\mathrm{S}$ bonds [25]. These FT-IR assignments are further clearly confirmed by XPS analysis (Fig. 1E). Representative peaks of C 1s, N 1s, 01 s, and S 2p are observed at 283, 398, 529, $161 \mathrm{eV}$, respectively [26], indicating that the NIR-CDs mainly contain C, N, O and S elements (atom ratio, C:O:N:S = 
63.9:16.3:19.4:0.4). High resolution C 1s spectrum (Additional files Fig. S2a) reveals four typical peaks at 284.8, 286.3, 288.0 and 290.1, which is attributed to $\mathrm{C}=\mathrm{C} / \mathrm{C}-\mathrm{C}, \mathrm{C}-\mathrm{N} / \mathrm{C}-\mathrm{O}, \mathrm{C}=\mathrm{N} / \mathrm{C}=\mathrm{O}$ and $\mathrm{N}-\mathrm{C}=0$, respectively [27]. Three obvious peaks of pyridine-like $\mathrm{N}$, graphitic $\mathrm{N}$, and pyrrole-like $\mathrm{N}$ at 398.5, 400.0, and $402.6 \mathrm{eV}$ are severally observed in high resolution $\mathrm{N}$ 1s spectrum (Additional files Fig. S2b) [28]. $\mathrm{O}$ 1s XPS spectrum shows two unique peaks of $\mathrm{C}-\mathrm{OH}$ and $\mathrm{C}=0$ at 531.3 and $533.6 \mathrm{eV}$ [29], respectively (Additional files Fig. S2c). Besides, in the high solution S 2p spectrum, four binding energies at $162.2,163.4,164.8$ and 169.7 were discriminated, corresponding to thiolate, $2 \mathrm{p}_{3 / 2}$ and $2 \mathrm{p}_{1 / 2}$ of thiophene $S$, and oxidized S, respectively [30]. Finally, zeta potential measurement reveals that the NIR-CDs are negatively charged ( $\zeta=-19.2 \mathrm{mV}$, Additional files Fig. S3), which brings strong electrostatic exclusion and good colloid stability.

Thereafter, the photophysical properties including Uv-vis absorption, fluorescence spectrum and fluorescence lifetime of the NIR-CDs were inspected, respectively. As shown in Fig. 2A, three distinct absorption bands i.e., 240-300 nm, 350-450 nm, and 550-750 nm are observed, which are generally ascribed to the typical $\pi \rightarrow \pi^{*}$ transition of the aromatic $\mathrm{C}=\mathrm{C}$ bond, $\pi \rightarrow \pi^{*}$ and $\mathrm{n} \rightarrow \pi^{*}$ transitions of the aromatic $\pi$ system containing $\mathrm{C}=\mathrm{O}, \mathrm{C}=\mathrm{N}$, and $\mathrm{C}=\mathrm{S}$ bonds [25, 31, 32], respectively. In Fig. 2b, the NIR-CDs reveal strongly deep-red emission from 625 to $725 \mathrm{~nm}$ with a sharp peak centered at $680 \mathrm{~nm}$ under different excitation wavelengths. Such an excitation-independent fluorescence emission property is usually attributed to the surface states/defects induced emission [33]. In addition, the average fluorescence lifetime of the NIR-CDs is measured and calculated to be $3.2 \mathrm{~ns}$ with bi-exponential decays (Additional files Fig. S4), and the absolute fluorescence quantum yield is measured to be $18.4 \%$ under an optimal excitation of $420 \mathrm{~nm}$. In our previous reports $[21,23]$, the NIR-CDs have been demonstrated excellent tolerance to photobleaching under the excitation of ultraviolet light, good storage stability at ambient environment and competitive agent in light-harvesting and electron transfer from photosystem II (PS II) to photosystem I (PS I) in chloroplasts [18, 34].

\section{NIR-CDs treatment significantly improved growth and photosynthesis of T. hemsleyanum}

Figure $3 \mathrm{~A}$ is the phenotypic characteristic of $T$. hemsleyanum exposed to $0.05 \mathrm{mg} / \mathrm{mL}$ NIR-CDs on day 30 . The treatment enables T. hemsleyanum to grow more exuberantly than the control group. As shown in Fig. 3B, compared to the control, root length, stem length, leaf area, net photosynthetic rate, stomatal conductance, transpiration rate, chlorophyll fluorescence parameter Fv/Fn and chlorophyll content were significantly increased by $38.36 \pm 6.6 \%, 41.40 \pm$ $1.9 \%, 93.84 \pm 9.2 \%, 16.25 \pm 1.2 \%, 122.51 \pm 10.6 \%, 120.14 \pm 11.1 \%, 80.36 \pm 7.1 \%$ and $87.34 \pm 3.2 \%$, respectively. Intercellular $\mathrm{CO}_{2}$ was decreased by $51.93 \pm 3.8 \%$ than the control.

Confocal images were captured using Laser-scanning confocal fluorescence microscope after incubation with 0.05 $\mathrm{mg} / \mathrm{mL}$ NIR-CDs. As shown in Fig. 3A, the red fluorescence signals from NIR-CDs observed under $514 \mathrm{~nm}$ excitation were widely distributed in root, stem, and leaf of T. hemsleyanum, which confirmed that NIR-CDs could penetrate cell wall into vascular bundle system, and were transported to the aerial parts.

\section{Metabolome profiling and KEGG enrichment analysis of differential metabolites}

We profiled the widely-targeted LC-MS/MS based metabolome of the samples from the control (denoted as CK) and the NIR-CDs-treatment groups (denoted as TH), which represented the impact of the NIR-CDs on metabolite accumulation of T. hemsleyanum. We detected 493 secondary metabolites grouped into 8 classes (Additional files Table S1), including 182 flavonoids, 167 phenolic acids, 20 lignans and coumarins, 64 alkaloids, 15 terpenoids, 26 tannins and others. The differentially accumulated metabolites (DAM) between the control and the experimental groups (CK_vs_TH) were screened using the variable importance in projection (VIP) $\geq 1$ from the OPLS-DA model and fold change $\geq 1.5$ (upregulated) or $\leq 0.667$ (downregulated). A total of 191 DAM were identified in CK_Vs_TH (Additional files Table S2). These secondary metabolites can be mainly categorized into the classes of flavonoids and 
phenolic acids, including 106 flavonoids, 43 phenolic acids, 12 lignans and coumarins, 9 alkaloids, and others. Overall, flavonoids were more inclined to accumulate in $\mathrm{CK}$ than in $\mathrm{TH}$. The content of phenolic acids, lignans and coumarins were significantly improved by CDs-treatment (Fig. 4A).

We focused on the two classes of secondary metabolites (flavonoids and phenolic acids) likely to be major contributors to biological activity (Fig. 4B). Flavonols and flavonoid carbonoside were identified with a series of glycoside derivatives of kaempferol, quercetin, and apigenin, which made up the majority of the DAMs detected for CK _Vs_TH compared samples. Based on fold changes and VIP values, 77 out of 106 flavonoids were identified as downaccumulated significantly by CDs-treatment, the concentrations of Kaempferol-3-0-glucoside, Quercetin-3-0neohesperidoside, Apigenin-6,8-di-C-glucoside-4'-0-glucoside, Quercetin-3-0-sophoroside-7-0-rhamnoside, Procyanidin $\mathrm{C} 1$, and Epigallocatechin were significantly greater in $\mathrm{CK}$ than in $\mathrm{TH}$ (Student's t test, $P<0.05$ ), and the concentrations of the remaining 29 flavonoids were significantly greater in TH than in CK. 43 out of 167 phenolic acids were identified as differentially accumulated by CDs-treatment, of these 13 phenolic acids were downregulated and 30 were upregulated in TH compared with CK, among which Quillaic acid, Furanofructosyl-a-D-(6-mustard acyl)glucoside, Feruloylcaffeoyltartaric acid were found only in TH, CDs-treatment also led to a 10-fold enhancement in Syringin, Neochlorogenic acid, Homogentisic acid, and 5-fold increase in 1-Caffeoylquinic acid and 2-Caffeoylquinic acid.

\section{Transcriptome analysis and DEGs Identification}

Differentially expressed genes (DEGs) in CK vs TH comparative group were identified by a transcriptomic comparison. A total of 40.08-52.20 million clean reads were obtained, and the Q30 of the raw reads ranged from $91.08-92.21 \%$, indicating the high quality of the transcriptome data. As shown in Figure $3 \mathrm{~A}$, the red dots indicate the upregulated expressed genes (Log2FC $\geq 2$ ), the black dots indicate the non-significantly differentially expressed genes $(0.5<$ Log2FC $<2$ ), and the blue dots indicate the downregulated expressed genes (fold change, Log2FC $\leq 0.5$ ). The horizontal axis indicates the fold change of differential expression, and the vertical axis indicates the significance level of the gene expression differences. According to the results of transcriptome data, 43340 unigenes were identified. A total of 6,874 DEGs were identified between CK vs TH (Additional files Table S3), including 2962 upregulated genes and 3912 downregulated genes (Fig. 5A).

The DEGs between CK and TH were subjected to KEGG, KOG, and GO functional pathway analyses. The top enriched KEGG terms contributed by these DEGs were ko01110 (Biosynthesis of secondary metabolites), ko04016 (MAPK signaling pathway - plant), ko00941 (Flavonoid biosynthesis), ko00860(Porphyrin and chlorophyll metabolism), and ko00909 (Sesquiterpenoid and triterpenoid biosynthesis) (Fig. 5B). The top enriched KOG terms included Posttranslational modification, protein turnover, chaperones(O), Carbohydrate transport and metabolism(G), Energy production and conversion(C), and Secondary metabolites biosynthesis, transport and catabolism(Q) (Fig. 5C). DEGs annotated in GO were classified into 52 functional groups, including 25 groups in biological process, 16 in cellular components, and 11 in molecular function. 'Cell', 'Cell part', and 'organelle' were the terms that dominated in the cellular component category. In the 'molecular function' category, the GO terms 'catalytic activity' and 'binding' predominated. 'Cellular process', 'metabolic process', 'biological regulation', and 'regulation of biological process' were the most represented GO terms in the biological process category (Fig. 5D). The above functional pathway analyses indicated that most of the identified DEGs acted on metabolic processes related to secondary metabolite metabolism and carbohydrate metabolism.

\section{Co-expression network analysis of DEGs}

To identify the candidate genes regulating secondary metabolite metabolism and carbohydrate metabolism, an effective system biology method called weighted gene co-expression network analysis (WGCNA), was performed to find the modules of highly correlated genes and relate these modules to traits. Modules are clusters of genes with high 
correlation, and genes of a same module are co-expressed. A total of 8 gene modules were established on the clustering and signature analysis of the genes with similar expression patterns in DEGs (Additional files: Fig. S5). They were then used to correlate with the traits of flavonoid metabolites content (Additional files: Fig. S6), phenolic acids metabolites content (Additional files: Fig. S7), and photosynthetic efficiency (Additional files: Fig. S8), respectively. Among the 8 modules, the modules of "darkturquoise" and "green" were found to be associated with flavonoid, and phenolic acids accumulation. The "darkturquoise" gene module showed a strong correlation with the concentration of flavonoids, such as isorhamnetin-3-0-gallate (neohesperidin) $(r=1.0, P<0.01)$, Quercetin-4'-0-glucuronide $(r=0.98, P<$ 0.01), Dihydrokaempferol-3-0-glucoside ( $r=0.98, P<0.01)$, Quercetin-3-0-(2"-0-galactosyl) glucoside $(r=0.98, P<$ 0.01), Dihydrokaempferol-3-0-glucoside $(r=0.98, P<0.01)$, Luteolin-7-0-glucoside (Cynaroside) $(r=0.98, P<0.01)$, Kaempferol-3-0-sulfonate $(r=0.92, P<0.01)$, Apigenin-7-0-rutinoside (Isorhoifolin) $(r=0.97, P<0.01)$, and so on. The "green" gene module also showed a high positive correlation with flavonoids, such as Kaempferol-3-0-glucoside (Astragalin) ( $r=0.95, P<0.01)$, Luteolin-8-C-glucoside (Orientin) $(r=0.98, P<0.01)$, Apigenin-8-C-Arabinoside $(r=0.94$, $P<0.05)$, and so on. The "darkturquoise" gene module showed a strong correlation with the concentration of phenolic acids, such as Syringin ( $r=1.0, P<0.01)$, 3,4-Dihydroxybenzeneacetic acid $(r=0.99, P<0.01)$, Sinapaldehyde-4-0Glucoside ( $r=0.99, P<0.01), 2$-0-Caffeoylmalic acid $(r=0.93, P<0.01)$, Neochlorogenic acid $(r=0.97, P<0.01)$, Homogentisic acid $(r=0.94, P<0.01)$, and so on.

The module-trait relationship analysis also identified module "darkturquoise" as most highly related to photosynthetic efficiency. Photosynthetic rate $(\mathrm{Pn})(\mathrm{r}=0.97, P<0.01)$, stomatal conductance (Cond) $(r=0.96, P<0.01)$, transpiration rate $(\mathrm{Tr})(\mathrm{r}=0.99, P<0.01)$, chlorophyll fluorescence parameters (Fv/Fm) $(r=0.98, P<0.01)$. Therefore, the "darkturquoise" and "green" modules were selected as the key gene modules for subsequent analysis.

\section{Key gene module expression and functional analysis}

Gene annotation and expression of all the gene members in the two key gene modules were performed. "darkturquoise" and "green"gene modules were composed of 6596 and 940 unigenes, respectively. When the differential genes of CK vs TH were separately intersected with the gene members of the two key gene modules, we found that the intersection was observed between the two key gene modules and the differentially expressed genes (Fig. 6A). Downregulated expressed genes in "darkturquoise" and "green" gene modules were 985 and 83, while upregulated expressed genes in the two modules were 1621 and 44, respectively. Further expression analysis of gene members of the two key gene modules revealed distinct expression patterns in CK and TH (Fig. 6B and 6C).

The KEGG gene-set enrichment analysis of these DEGs in "green" and "darkturquoise" gene modules were severally shown as Fig. 7 and Fig. 8, respectively. For each screened gene module, the correlation scatter plot between some phenotypes and modules were drawn (Fig. 7A and Fig. 8A). The enriched KEGG terms of upregulated expressed genes in "green" gene module contained MAPK signaling pathway, porphyrin and chlorophyll metabolism, and galactose metabolism (Fig. 7B). The enriched KEGG terms of downregulated expressed genes in "green" gene module contained flavonoid biosynthesis, other glycan degradation, glycosaminoglycan degradation (Fig. 7C). The top enriched KEGG terms of upregulated expressed genes in "darkturquoise" gene module were benzoxazinoid biosynthesis, MAPK signaling pathway, porphyrin and chlorophyll metabolism, and anthocyanin biosynthesis (Fig. 8B). The top enriched KEGG terms of downregulated expressed genes in "darkturquoise" gene module were flavonoid biosynthesis, biosynthesis of secondary metabolites (Fig. 8C).

\section{Identification of hub genes within network modules}

Two modules, "darkturquoise" and "green", were considered to take a decisive factor in the regulation of CDs on plant growth and secondary metabolites accumulation. Hub genes were defined as these genes which were highly associated with other genes in each module network, and played a central role within the network clusters. Hub genes 
within the two modules were discovered in Fig. 9. Protein-protein interaction (PPI) network, which took the gene members in the two key modules as its object, were obtained using string database, and a total of 44 nodes, 44 edges were identified (Fig. 9A). The MCC algorithm of CytoHubba was used to score and sort the hub nodes in the PPI network, and select the top 5 genes (triosephosphate isomerase, mitochondrial carrier protein, thymidylate kinase, dehydrogenase E1 component and lyase) as the hub genes of the PPI network (Fig. 9B). Gene expression of all PPI network gene members in the two key gene modules were performed and shown in Fig. 9C. Among them, $17 \mathrm{up}-$ regulated genes and 27 down-regulated genes were identified. These interactions among key genes, pathways, metabolic type and gene regulation are shown in Fig. 9D. As for secondary metabolism, transferase family genes in anthocyanin, phenylpropanoid, and flavonoid biosynthesis pathways tended to be down-regulated after CDs-treatment, and the expression of NAD dependent epimerase/dehydratase family genes in phenylpropanoid, flavonoid, and isoflavonoid biosynthesis pathways were restrained as well. As for primary metabolism, some gene families of carbohydrate metabolism, such as pfkB family carbohydrate kinase, glycosyl hydrolase family, triosephosphate isomerase, and carbohydrate phosphorylase, tended to be down-regulated after CDs-treatment. However, some gene families involved in carbohydrate synthesis, such as sucrose synthase and aldehyde dehydrogenase families, were upregulated. Ferrochelatase and chlorophyll A-B binding protein, which were considered to be vital components of photosynthesis and chloroplast synthesis, were both up-regulated in response to CDs-treatment.

\section{Validation of hub genes expression by qRT-PCR}

We selected the top 5 hub genes (triosephosphate isomerase, mitochondrial carrier protein, thymidylate kinase, dehydrogenase E1 component and lyase) of the PPI network to conduct a validation experiment by qRT-PCR analysis. The patterns of RNA-Seq expressions on all the 5 hub genes were highly consistent with the qRT-PCR data, and a correlation coefficient (R2) of $95.33 \%$ was obtained (Fig. 10). Both qRT-PCR and RNA-seq analyses showed that three hub genes (triosephosphate isomerase, mitochondrial carrier protein, and dehydrogenase E1 component) showed lower level of expression after CDs-treatment. However, other two genes, thymidylate kinase and lyase showed a higher level of expression in the CDs-treatment group.

\section{Discussion}

As an emerging research hotspot in nanotechnology, carbon dots (CDs) have attracted much attention. Due to the fluorescence property of CDs, it is available to trace the uptake and accumulation of CDs in plants. When the mung bean was treated with nitrogen-doped CDs by solid-state method, the blue fluorescence was observed to migrate from the beans to the root ends by ultraviolet light during the sprouting process [35]. When T. hemsleyanum was treated with red CDs, the fluorescence signal of CDs was mainly detected in the vascular system of the root, stem, and leaf by confocal laser scanning microscope in our study, which confirmed that CDs could easily cross the biological barrier and be widely distributed in the plants. A number of studies have demonstrated that CDs can promote the growth of various plants by enhancing the light absorption [11], stimulating biosynthase activity [36], converting ultraviolet light into blue and red light $[17,18]$, and we firstly demonstrated that potential molecular mechanisms behind the growthstimulating effect might be related to up-regulated expression of the primary metabolism related genes, among which PsbP and PsiKgenes were the hub genes [21].

Plants synthesize a myriad of secondary metabolites that are derived from central or primary metabolism. Flavonoids, alkaloids, terpenoids and phenolic acids are the common and important kinds of secondary metabolites, and they are closely related to multiple bioactive functions of medicinal plant. It is generally assumed that the level of primary metabolism would have a major positive impact on the growth rate of plant and the level of secondary metabolism would be stimulated under environmental stress [37]. Hence, it is of great significance to investigate the potential impact of CDs on the metabolic transition between primary and secondary metabolism.

Page $7 / 25$ 
T.hemsleyanum is a herbal plant distributed in tropical to subtropical regions, mainly in mainland China. Flavonoids and phenolic acids were the main active secondary metabolic ingredients of T. hemsleyanum [38]. Total favonoids from T. hemsleyanumon were reported to exert anti-infammatory effects on Con A-induced hepatitis in mice [39]. T. hemsleyanum flavonoids could inhibit the migration and promote the apoptosis of A549 cells both in vitro and in vivo [40]. Phenolics in T. hemsleyanum, including 1-Caffeoylquinic acid, 2-Caffeoylquinic acid, 5-p-coumaroylquinic acid, isoorientin etc., were related to the antioxidant and antiproliferative activities of T. hemsleyanum [41]. A total of 13 bioactive compounds, including kaempferol, caffeic acid, apigenin, quercetin etc., were considered to be related to alleviating lung infection in Pseudomonas aeruginosa-induced mice [42]. Apigenin and luteolin glycosides of $T$. hemsleyanum were reported to induce apoptosis in HepG2 cells as well as inhibit the tumor growth in $\mathrm{H} 22$ tumorbearing mice [43].

In this study, CDs-treatment had the opposite effect on the content of phenolic acids and flavonoids. The contents of flavonoids decreased and phenolic acids substance increased. The ingredients associated with anti-inflammatory and antioxidant activities of T. hemsleyanum, such as syringin, neochlorogenic acid, homogentisic acid, 1-Caffeoylquinic acid and 2-Caffeoylquinic acid, were generally improved in response to CDs-treatment. But the ingredients associated with anti-tumor activity, such as glycoside derivatives of kaempferol, quercetin, and apigenin, were more likely to decrease in general terms. Thereafter, we carried out an integrated transcriptome and metabolome research on the regulatory networks of primary and secondary metabolites biosynthesis, including starch and sucrose metabolism, photosynthesis, flavonoids biosynthesis etc. of T. hemsleyanum under the treatment of NIR-CDs. The pattern of association between differentially expressed genes (DEGs) and metabolite components were explored by WGCNA, then 44 candidate hub genes highly related to quality ingredients accumulation and growth were identified.

Maintenance of deoxyribonucleotide levels is crucial for ensuring efficiency and genome stability of DNA replication and DNA synthesis. The increase in the deoxyribonucleotide pools would lead to subtle changes in the function of DNA Pol III leading to more elongation and less proof-reading mode, thus resulting in the activation of translesion synthesis. Thymidylate kinase, referred to as TMK or TMPK in different organisms, is an important enzyme in DNA biosynthesis and catalyses the conversion of dTMP to dTDP. In the present study, the activity of thymidylate kinase was significantly stimulated by NIR-CDs-treatment, serving as one of the top 5 hub genes. It suggested that thymidylate kinase gene was highly associated with other genes in DNA synthesis and cell division gene regulatory network, and played a central role in promoting primary metabolism of $T$. hemsleyanum. Thymidylate kinase has also been studied in the photosynthetic nitrogen-fixing cyanobacterium Nostoc. Recombinant Nostoc strains overexpressing AnTMK exhibited higher growth rate measured in terms of chlorophyll a content under normal growth conditions, which indicated that the TMK is likely to have a significant role in photosynthetic organisms [44].

Mitochondrial carrier family (MCF) proteins of plant play a major role in transporting TCA-cycle intermediates across the inner mitochondrial membrane [45]. The present study also suggested MCF served as a protagonist in the regulation of mitochondrial metabolism in response to CDs-treatment. Chlorophyll and chlorophyll A-B binding protein are indispensable for the assembly of a functional photosystem II, which is responsible for light absorption, excitation energy transfer, and charge separation. Ferrochelatase in plants possesses a conserved transmembrane chlorophyll A$\mathrm{B}$ binding domain that is somewhat analogous to the first and the third helix of light-harvesting complexes, including a chlorophyll-binding motif [46]. In the present study, CDs-treatment significantly stimulated photosynthesis of $T$. hemsleyanum by simultaneously upregulating the expression of chlorophyll A-B binding protein and ferrochelatase.

Glycosyl hydrolase family genes participated in many aspects of plant physiological processes, in particular biotic and abiotic stresses through the regulation of phytohormones and defensive components. Four genes of the glycoside hydrolase family 1 in the model legume plant Medicago truncatula, were dramatically activated by $\mathrm{NaCl}, \mathrm{PEG}, \mathrm{IAA}$,

Page 8/25 
ABA, SA and GA3 treatments [47]. Both triosephosphate isomerase and pfkB family carbohydrate kinase are key enzymes in glucolysis [48]. Sucrose synthase plays an important role in tricarboxylic acid cycle and polysaccharides synthesis. In the present study, CDs-treatment significantly affected glucose metabolism by simultaneously inhibiting triosephosphate isomerase and pfkB family carbohydrate kinase, while activating sucrose synthase, which suggested that CDs could switch the glycometabolism flow from the glucolysis pathway to the pyruvate carboxylation, and hence the generation of polysaccharide would be improved.

Transferases are a superfamily of abundant enzymes that play vital roles in plant growth and development and flavonoid metabolism. For example, UDP-glycosyltransferases were essential for flavonoid biosynthesis in Glycyrrhiza uralensis [49]. A glutathione $S$ transferase gene was demonstrated to be a candidate gene for manipulating anthocyanin accumulation and pigmentation in cotton tissues [50]. Transferases are likely to be responsive to exogenous chemical ingredients or environmental stress, and thus the accumulation of plant-derived flavonoids was affected. The glutathione transferases had members that are selectively induced by chemical stress treatments, which were confirmed to have roles in redox homeostasis and maintaining the flavonoid pool under stress conditions [51].

\section{Conclusion}

In summary, UV-absorptive and water-soluble NIR-CDs were harvested via a facile microwave-assisted carbonization method. The NIR-CDs were successfully adopted for enhancing photosynthesis and growth of T. hemsleyanum, and the influences on the accumulation of bioactive ingredients was illustrated as well. A total of 191 differential secondary metabolites and 6874 differentially expressed genes were identified. The phenolic acids were generally improved, but the flavonoids were more likely to decrease in general terms. The pivotal differentially expressed genes were involved in multiple metabolic processes related to biosynthesis of secondary metabolites, flavonoid biosynthesis, porphyrin and chlorophyll metabolism, etc. Subsequently, DEGs were divided into 9 modules by WGCNA. Two modules positively correlated with the flavonoids content, phenolic acids content and photosynthetic efficiency were identified, in which 44 hub genes and the top 5 genes of the PPI network were identified. This research provided key regulatory genes and differentially accumulating quality ingredients under CDs-treatment, which could provide a theoretical basis for expanding the applications of nanomaterial in industrial crop agriculture.

\section{Materials And Methods}

\section{Materials and apparatus}

Reduced glutathione (GSH), $\mathrm{Na}_{2} \mathrm{HPO}_{4}, \mathrm{KH}_{2} \mathrm{PO}_{4}$ and $\mathrm{KCl}$ were purchased from Aladdin Chemistry Co., Ltd (Shanghai, China). Formamide was obtained from Sinopharm Chemical Reagent Co., Ltd. (Shanghai, China). All of the chemicals were used as received without further purification. Aqueous solutions were prepared using deionized (DI) water.

A microwave oven (Galanz, P70F20CL) was adopted for the fabrication of NIR-CDs. The sizes and morphologies of the obtained NIR-CDs were characterized by high resolution transmission electron microscopy (HR-TEM, Tecnai F20) using an acceleration voltage of $200 \mathrm{kV}$. Fourier transform infrared (FT-IR) spectrum was carried out on a Nicolet 6700 FT-IR spectrometer through the $\mathrm{KBr}$ pellet technology. Raman spectrum was recorded on a Renishaw inVia Raman spectrophotometer via a $532 \mathrm{~nm}$ laser as the excitation resource. The crystal phase of NIR-CDs was measured on a Bruker D8 Discover X-ray diffractometer (XRD) with $2 \theta$ range from $10^{\circ}$ to $50^{\circ}$ at a scanning rate of $4^{\circ} / \mathrm{min}$, with $\mathrm{Cu} \mathrm{Ka}$ irradiation $(k=1.5406 \AA$ ). Fluorescence spectra were measured on a Perkin Elmer spectrophotometer (LS-55). UV-vis absorption spectra were recorded on an Agilent Cary 300 spectrophotometer. Fluorescence delay curve was monitored 
by Fluorolog 3-11 (HORIBA Jobin Yvon). Absolute fluorescence quantum yield (QY) was directly measured by a Fluoromax-4 measurement system (HORIBA, JobinYvon. Inc).

\section{Synthesis of NIR-CDs}

NIR-CDs were synthesized in accordance with our previously-reported one-pot microwave-assisted carbonization manner [21, 52]. Briefly, reduced GSH $(2.0 \mathrm{~g})$ was dissolved in $20 \mathrm{~mL}$ formamide under a 10 mins treatment of ultrasonic. Thereafter, the mixture was transferred into a domestic microwave oven for further carbonization reaction (700 W, 3 mins). Then, the beaker was cooled down to room temperature naturally, and a dark green mixture was obtained. The mixture was centrifuged at $10000 \mathrm{rpm}$ for $5 \mathrm{~min}$ to remove large-sized nanoparticles, and purified through dialysis (cut-off molecular weight, 3500) for 5 days. Then, the NIR-CD s solution was concentrated and dried using a rotary evaporation. Finally, the dark green NIR-CDs powder ca. $350 \mathrm{mg}$ was harvested.

\section{Plant cultivation and NIRCDs treatment}

Healthy cutting seedlings of $T$. hemsleyanum were cultured in a greenhouse under a 16/8 h photoperiod (day/night), $23 \pm 2{ }^{\circ} \mathrm{C}$ temperature, and $50 \%$ relative humidity. Then, seedlings showing consistent growth state were selected and uniformly divided into two groups, CK_and_TH. NIR-CDs solutions at $0.05 \mathrm{mg} / \mathrm{mL}$ were sprayed on seedlings with a dosage of $10 \mathrm{~mL} /$ pot every day $(\mathrm{TH})$, and the same volume of ultrapure water was sprayed as the control (CK). Phenotypic changes were observed and recorded every 15 days, respectively. The photosynthetic parameters were determined according to our previous report [53]. Each treatment was repeated three times with ten plants. The young leaves were collected on 30 nd day, frozen in liquid nitrogen immediately, and stored at $-80^{\circ} \mathrm{C}$ for RNA-seq and metabolomics analysis. Three biological replicates were performed, and each biological replicate consisted of a pool of samples from 10 seedlings.

\section{RNA-seq data analysis}

RNA extraction, quantification and transcriptome sequencing were performed according to previous studies $[54,55]$. Then, sequencing libraries were generated using NEBNext ${ }^{\circledR}$ UltraTM RNA Library Prep Kit for Illumina ${ }^{\circledR}$ (NEB, USA). The clustering of the index-coded samples was performed on a cBot Cluster Generation System using TruSeq PE Cluster Kit v3-cBot-HS (Illumia). The library preparations were sequenced on an Illumina Hiseq platform and 125 bp/150 bp paired-end reads were generated. Transcriptome De novo assembly was performed using Trinity software. DIAMOND BLASTX software was used to compare the assembled transcript sequence with KEGG, NR, Swiss-Prot, GO, KOG, Trembl databases [56]. After predicting the amino acid sequence of the transcript, use HMMER software to compare with Pfam database to obtain the annotation information of the transcript. The data from RNA-seq were sequentially processed, after which gene expression quantity calculations were performed by bowtie2 [57], resulting in an expression matrix. Fragments per kilobase of transcript per million mapped reads (FPKM) of each gene based on the gene length were used to estimate the transcription or gene expression level.

The differential expression between the two groups was performed using edgeR. The corrected $\mathrm{P}$ value and |log2foldchangel are used as the threshold for significant difference expression (log2|FoldChange| $>2$ and $\mathrm{P}$ value $<$ 0.05 were adopted as the screening threshold). The enrichment analysis is performed based on the hypergeometric test. Kyoto Encyclopedia of Genes and Genomes (KEGG) were used as pathway enrichment. At least three genes were enriched in pathways, and a p value less than 0.05 was considered to be significantly enriched.

\section{Extraction, qualitative and quantitative analysis of metabolites}

Dissolve $100 \mathrm{mg}$ of freeze-dried sample powder with $1.2 \mathrm{~mL} 70 \%$ methanol solution, the mixture was extracted overnight at $4^{\circ} \mathrm{C}$ and then analyzed using an UPLC-ESI-MS/MS system (UPLC, SHIMADZU Nexera X2, 
https://www.shimadzu.com.cn/; MS, Applied Biosystems 4500 Q TRAP, https://www.thermofisher.cn/cn/zh/home/ brands/applied-biosystems.html). UPLC column was Agilent SB-C18 (1.8 $\mu \mathrm{m}, 2.1 \mathrm{~mm} \star 100 \mathrm{~mm})$. The mobile solvent system was consisted of solvent $A$, water ( $0.1 \%$ formic acid), and solvent $B$, acetonitrile $(0.1 \%$ formic acid). The gradient program was $95: 5 \mathrm{~V} / \mathrm{V}$ at $0.0 \mathrm{~min}$, a linear gradient to $5 \% \mathrm{~A}, 95 \% \mathrm{~B}$ was programmed within $9 \mathrm{~min}$, and then 5 : $95 \mathrm{~V} / \mathrm{V}$ was kept for $1 \mathrm{~min}$. Flow rate and injection volume was $0.35 \mathrm{~mL} / \mathrm{min}$ and $4 \mu \mathrm{L}$, respectively. The effluent was alternatively connected to an ESI-triple quadrupole-linear ion trap (QTRAP)-MS.

Linear ion trap (LIT) and triple quadrupole (QQQ) scans were acquired on an AB4500 Q TRAP UPLC/MS/MS System, equipped with an ESI Turbo Ion-Spray interface, and controlled by Analyst 1.6.3 software (AB Sciex). The ESI source operation parameters were as follows: ion source, turbo spray; ion spray voltage (IS) $5500 \mathrm{~V}$ (positive ion mode)/-4500 $\mathrm{V}$ (negative ion mode); source temperature $550^{\circ} \mathrm{C}$; ion source gas I (GSI), gas II(GSII) and curtain gas (CUR) were fixed at 50,60 , and $25.0 \mathrm{psi}$, respectively. Instrument tuning and mass calibration were performed with 10 and $100 \mu \mathrm{mol} / \mathrm{L}$ polypropylene glycol solutions in QQQ and LIT modes, respectively.

Metabolites were identified from the $\mathrm{m} / \mathrm{z}$ values, retention time, and fragmentation patterns of the standards in a database of MetWare Biotechnology Co., Ltd. (Wuhan, China). The filtering conditions for significantly changed metabolites were determined by VIP $\geq 1$ and Log2FC (fold change) $\geq 1$. VIP values were extracted from OPLS-DA result.

\section{Weighted gene co-expression network analysis (WGCNA)}

Weighted gene co-expression network analysis (WGCNA) is a systems biology method used to describe the gene association patterns between different samples. It can be used to identify highly synergistic gene sets, and identify functional gene sets that affect or participate in phenotypes according to the interconnection of gene sets and the association between gene sets and phenotypes [58]. R software WGCNA was used to construct an undirected cocorrelation network, defining a soft threshold $\beta=1$ for the screening condition. The minimum number of module genes was 30 and the module merging threshold was 0.25 , the cluster tree was constructed according to the correlation of gene expression and divided into modules. In order to identify gene modules and gene module members related to flavonoids and phenolic acid metabolism, the contents of net photosynthetic rate (Pn), stomatal conductance (Cond), transpiration rate (TR), intercellular carbon dioxide concentration (Cl), chlorophyll fluorescence parameters (Fv/FM), the trait matrix of related phenotypes were used to calculate the correlation between gene module and phenotype. The expression patterns of hub module gene members were quantitatively described by intramodular gene expression heatmap with gene significance analysis. The phenotype and gene significance of the screened gene modules were analyzed, and the Venn plot was used to describe the up-down relationship of differential genes and the correlation between phenotypic related module gene members, so as to determine the signal pathways and metabolic pathways that are inhibited or stimulated in KEGG enrichment analysis and identification of gene modules.

\section{Identification of hub genes associated with secondary metabolite synthesis}

Using the functionally annotated differential transcript data, the screened modules related to phenotype are combined to generate node information for constructing protein-protein network. Then, the near source species of $T$. hemsleyanum was selected for protein-protein interaction (PPI) analysis through STRING database (https://www.string-db.org/). Use Cytoscape software to draw the PPI network, then use the MCC algorithm of CytoHubba plug-in to score and sort the key nodes in the PPI network, and select the top 5 genes as the hub genes of the PPI network. Then, through the cluster analysis of the expression pattern of node genes in PPI network, the 
expression heatmap of these network member genes was drawn. Finally, the gene information in this network was described by sanky plot.

\section{Validation of the DEGs data using qRT-PCR}

Expression levels of selected hub genes were analyzed by Quantitative real-time PCR (qRT-PCR) using CFX96 real-time PCR system (BIO-RAD, USA). Total RNA of T. hemsleyanum was isolated using Plant Total RNA Isolation Kit (Sangon, Shanghai, China). cDNA synthesis was performed with TransScript ${ }^{\circledR}$ Reverse Transcriptase (TransGen, Beijing, China). Each PCR reaction mixture contained $10 \mu \mathrm{l}$ of 2x Power SYBR green PCR master mix (Applied Biosystems, Forster City, CA, USA), $2 \mu \mathrm{l}$ of template CDNA, $2 \mu \mathrm{l}$ of forward and reverse primers (50 pmol) in a final volume of $20 \mu \mathrm{l}$ with three technical replicates of each gene. The PCR reactions were conducted by incubation at $95^{\circ} \mathrm{C}$ for 3 min followed by 40 cycles of $95^{\circ} \mathrm{C}$ for $15 \mathrm{~s}$ and $60^{\circ} \mathrm{C}$ for $40 \mathrm{~s}$. The relative expression of each gene was calculated after being normalized to that of actin gene. Transcripts were calculated by the $2^{-\Delta \Delta C t}$ method. Real-time primers were presented in Table 1. Each gene was tested in triplicate with three biological replicates. The data represents the mean \pm SD of three independent experiments.

Table 1 List of primer sequences of top hub genes used for qRT-PCR analysis

\begin{tabular}{llllll} 
Gene annotation & Gene ID & Primer F $\left(5^{\prime}-3^{\prime}\right)$ & Primer R $\left(5^{\prime}{ }^{\prime} 3^{\prime}\right)$ & Tm & Length(bp) \\
\hline Lyase & $\begin{array}{l}\text { Cluster- } \\
9600.25525\end{array}$ & AGAGGTTCCACAGTTCAG & CAAGCACCTTAGCAATCTC & 60 & 182 \\
\hline $\begin{array}{l}\text { Mitochondrial } \\
\text { carrier protein }\end{array}$ & $\begin{array}{l}\text { Cluster- } \\
9600.54068\end{array}$ & GTGGTCTTCCTCTTCGTAT & TGGTAGGAGCAGGAGTAA & 60 & 123 \\
\hline $\begin{array}{l}\text { Thymidylate } \\
\text { kinase }\end{array}$ & $\begin{array}{l}\text { Cluster- } \\
9600.8465\end{array}$ & ACCGTTACTCCTATTCTGG & TCTCTGGTGGTATGTCAAG & 60 & 126 \\
\hline $\begin{array}{l}\text { Dehydrogenase } \\
\text { E1 component }\end{array}$ & $\begin{array}{l}\text { Cluster- } \\
9600.77806\end{array}$ & GCGAGAACAATCACTATGG & AGCATCCATACCATCTACC & 60 & 113 \\
\hline $\begin{array}{l}\text { Triosephosphate } \\
\text { isomerase }\end{array}$ & $\begin{array}{l}\text { Cluster- } \\
9600.42222\end{array}$ & TTAGCCTATGAGCCTGTG & ACTAACGGAACCTCCATAC & 60 & 156
\end{tabular}

\section{Declarations}

- Ethics approval and consent to participate

Not applicable

\section{- Consent for publication}

All Authors have agreed to submit it in its current form for consideration for publication in your journal.

\section{- Competing interests}

There are no conflicts to declare.

\section{- Funding}

This work was financially supported by the Major Science and Technology Projects of Breeding New Varieties of Agriculture in Zhejiang Province (No. 2021C02074), Zhejiang Provincial Natural Science Foundation (LY20B050003), 
Ningbo Science and Technology Bureau (2018B10054), Ningbo City Science and Technology Innovation 2025 Major Research Project (2019B10008), and the Ningbo 3315 Innovation Teams Program (2019A-14-C).

\section{- Authors' contributions}

Experiments were designed by MJL, JPZ, and YHW, and conducted by XP, ZMX, XHW, and ZYZ. Data was analysed by YXZ and CYY. Manuscript was prepared by XP and edited by YHW. All authors read and approved the final manuscript.

\section{- Acknowledgements}

Not applicable

\section{- Availability of data and material}

Complementary data of NIR-CDs are supplied as supplementary files Fig. S1- Fig. S4.

The metabolome and transcriptome original datasets generated in the current study are available in Table S1- Table s3.

Co-express network construction information generated in the current study are available in supplementary files Fig. S5- Fig. S8.

\section{References}

1. Jaganathan D, Ramasamy K, Sellamuthu G, Jayabalan S, Venkataraman G. CRISPR for crop improvement: an update review. Front Plant Sci. 2018;9: 985.

2. Glick BR. Plant growth-promoting bacteria: mechanisms and applications. Scientifica. 2012;2012: 963401.

3. Mickens MA, Torralba M, Robinson SA, Spencer LE, Romeyn MW, Massa GD, Wheeler RM. Growth of red pak choi under red and blue, supplemented white, and artificial sunlight provided by LEDs. Sci Hortic. 2019;245: 200.

4. Lin X, Li Y, Saravanakumar S, Tang Q, Zhang S, Gao X, Hu Y, Huang K, Han G. Sunlight-operable light converting smart windows for fertilizer-free plant growth enhancement. Nano Today. 2020;34: 100918.

5. Lowry GV, Avellan A, Gilbertson LM. Opportunities and challenges for nanotechnology in the agri-tech revolution. Nat Nanotech. 2019;14: 517.

6. Giraldo JP, Landry MP, Faltermeier SM, McNicholas TP, Iverson NM, Boghossian AA, Reuel NF, Hilmer AJ, Sen F, Brew JA, Strano MS. Plant nanobionics approach to augment photosynthesis and biochemical sensing. Nat Mater. 2014;13: 400.

7. Xiong JL, Li J, Wang HC, Zhang CL, Naeem MS. Fullerol improves seed germination, biomass accumulation, photosynthesis and antioxidant system in Brassica napus L. under water stress. Plant Physiol Biochem. 2018;129: 130.

8. Milenkovi I, Boriev M, Zhou Y, Spasi SZ, Radoti K. Leblanc RM. Photosynthesis Enhancement in Maize via Nontoxic Orange Carbon Dots. J Agric Food Chem. 2021;69: 5446.

9. Raliya R, Nair R, Chavalmane S, Wang WN, Biswas P. Mechanistic evaluation of translocation and physiological impact of titanium dioxide and zinc oxide nanoparticles on the tomato (Solanum lycopersicum L.) plant. Metallomics. 2015;7: 1584.

10. Wu H, Tito N, Giraldo JP. Anionic cerium oxide nanoparticles protect plant photosynthesis from abiotic stress by scavenging reactive oxygen species. ACS Nano. 2017;11: 11283-11297. 
11. Yu J, Yu H, Li L, Ni X, Song K, Wang L. Influence of luminescent nanomaterials on plant growth and development. ChemNanoMat. 2021;7: 859.

12. Alimohammadi M, Xu Y, Wang DY, Biris AS, Khodakovskaya MV. Physiological responses induced in tomato plants by a two-component nanostructural system composed of carbon nanotubes conjugated with quantum dots and in vivo multimodal detection. Nanotechnology. 2011;22: 8.

13. Nair R, Poulose AC, Nagaoka Y, Yoshida Y, Maekawa T, Kumar DS. Uptake of FITC labeled silica nanoparticles and quantum dots by rice seedlings: effects on seed germination and their potential as biolabels for plants. J Fluoresc. 2011;21: 2057.

14. Li Y, Li W, Zhang H, Liu Y, Ma L, Lei B. Amplified light harvesting for enhancing Italian lettuce photosynthesis using water soluble silicon quantum dots as artificial antennas. Nanoscale. 2020;12: 155.

15. Nordmann J, Buczka S, Voss B, Haase M, Mummenhoff K. In vivo analysis of the size- and time-dependent uptake of $\mathrm{NaYF}_{4}$ : Yb, Er upconversion nanocrystals by pumpkin seedlings. J. Mater. Chem. B 2015;3: 144.

16. Yin W, Zhou L, Ma Y, Tian G, Zhao J, Yan L, Zheng X, Zhang P, Yu J, Gu Z, Zhao Y. Phytotoxicity, Translocation, and Biotransformation of $\mathrm{NaYF}_{4}$ Upconversion Nanoparticles in a Soybean Plant. Small; 2015;11: 4774.

17. Li W, Wu S, Zhang H, Zhang X, Zhuang J, Hu C, Liu Y, Lei B, Ma L, Wang X. Enhanced biological photosynthetic efficiency using light-harvesting engineering with dual-emissive carbon dots. Adv Funct Mater. 2018;28: 1804004.

18. Li DN, Li W, Zhang HR, Zhang XJ, Zhuang JL, Liu YL, Hu CF, Lei BF. Far-Red carbon dots as efficient lightharvesting agent for enhanced photosynthesis. ACS Appl Mater Interfaces. 2020;12: 21009.

19. Xu X, Li W, Hu, C, Lei B, Zhang X, Li Y, Zhan Q, Liu Y, Zhuang J. Promoting the Growth of Mung Bean Plants through Uptake and Light Conversion of $\mathrm{NaYF}_{4}$ : Yb, Er@CDs Nanocomposites. ACS Sustainable. Chem. Eng. 2020;8: 9751.

20. Li Y, Xu X, Wu Y, Zhuang J, Zhang X, Zhang H, Lei B, Hu C, Liu Y. A review on the effects of carbon dots in plant systems. Mater Chem Front. 2020;4: 437.

21. Wang Y, Xie Z, Wang X, Peng X, Zheng J. Fluorescent carbon-dots enhance light harvesting and photosynthesis by overexpressing PsbP and PsiK genes. J Nanobiotechnol. 2021;19: 260.

22. Peng X, Luo YY, Wang J, Ji T, Yuan LX, Kai GY. Integrated analysis of the transcriptome, metabolome and analgesic effect provide insight into potential applications of different parts of Lindera aggregate. Food Res Int. 2020;138: 109799.

23. Nimi N, Saraswathy A, Nazeer SS, Francis N, Shenoy SJ, Jayasree RS. Multifunctional hybrid nanoconstruct of zerovalent iron and carbon dots for magnetic resonance angiography and optical imaging: An In vivo study. Biomaterials. 2018;171: 46.

24. Mewada A, Pandey S, Shinde S, Mishra N, Oza G, Thakur M, Sharon M, Sharon M. Green synthesis of biocompatible carbon dots using aqueous extract of Trapa bispinosa peel. Mater Sci Eng C. 2013;33: 2914.

25. Pan L, Sun S, Zhang L, Jiang K, Lin H. Near-infrared emissive carbon dots for two-photon fluorescence bioimaging. Nanoscale. 2016;8: 17350.

26. Travlou NA, Ginnakoudakis DA, Algarra M, Labella AM, Rodríguez-Castellón E, Bandosz TJ. S- and N-doped carbon quantum dots: Surface chemistry dependent antibacterial activity. Carbon. 2018;135: 104.

27. Sun S, Zhang L, Jiang K, Wu A, Lin H. Toward High-Efficient Red Emissive Carbon Dots: Facile Preparation, Unique Properties, and Applications as Multifunctional Theranostic Agents. Chem Mater. 2016;28: 8659.

28. Li H, Su D, Gao H, Yan X, Kong D, Jin R, Liu X, Wang C, Lu G. Design of Red Emissive Carbon Dots: Robust Performance for Analytical Applications in Pesticide Monitoring. Anal Chem. 2020;92, 3198. 
29. Ding H, Wei JS, Zhang P, Zhou ZY, Gao QY, Xiong HM. Solvent-Controlled Synthesis of Highly Luminescent Carbon Dots with a Wide Color Gamut and Narrowed Emission Peak Widths. Small. 2018;14, 1800612.

30. Bojdys MJ, Müller JO, Antonietti M, Thomas A. Ionothermal Synthesis of Crystalline, Condensed, Graphitic Carbon Nitride. Chem Eur J. 2008;14: 8177.

31. Liu J, Geng Y, Li D, Yao H, Huo Z, Li Y, Zhang K, Zhu S, Wei H, Xu W, Jiang J, Yang B. Deep red emissive carbonized polymer dots with unprecedented narrow full width at half maximum. Adv Mater. 2020;32: 1906641.

32. Ye X, Xiang Y, Wang Q, Li Z, Liu Z. A Red Emissive Two-Photon Fluorescence Probe Based on Carbon Dots for Intracellular pH Detection. Small. 2019;15: 1901673.

33. Zhu J, Sun S, Jiang K, Wang Y, Liu W, Lin H. A highly sensitive and selective fluorimetric probe for intracellular peroxynitrite based on photoinduced electron transfer from ferrocene to carbon dots. Biosens Bioelectron. 2017;97, 150.

34. Govorov AO. Enhanced Optical Properties of a Photosynthetic System Conjugated with Semiconductor Nanoparticles: The Role of Förster Transfer. Adv Mater. 2008;20: 4330.

35. Wang AW, Kang FW, Wang ZG, Shao QG, Li Z, Zhu GY, Lu J, Li YY. Facile Synthesis of Nitrogen-Rich Carbon Dots as Fertilizers for Mung Bean Spouts. Adv Sustain Syst. 2019;3: 1800132.

36. Zhang ML. Hu LL, Wang HB, Song YX, Liu Y, Li H, Shao MW, Huang H, Kang ZH. One-step hydrothermal synthesis of chiral carbon dots and their effects on mung bean plant growt. Nanoscale. 2018;10: 12734.

37. Rapp J, Rath P, Kilian J, Brilisauer K, Grond S, Forchhammer K. A bioactive molecule made by unusual salvage of radical SAM enzyme by-product 5-deoxyadenosine blurs the boundary of primary and secondary metabolism. J Biol Chem. 2021;296: 100621.

38. Ji T, Ji WW, Wang Juan, Chen HJ, Peng X, Cheng KJ, Qiu D, Yang WJ. A comprehensive review on traditional uses, chemical compositions, pharmacology properties and toxicology of Tetrastigma hemsleyanum. J Ethnopharmacol. 2021;264, 113247.

39. Ji WW, Peng X, Lou TL, Wang J, Qiu WY. Total favonoids from Tetrastigma hemsleyanum ameliorates infammatory stress in concanavalin A induced autoimmune hepatitis mice by regulating Treg/Th17 immune homeostasis. Inflammopharmacology. 2019;27: 1297.

40. Li YL, Feng XY, Yu X, Wang YX, Liu YY, Ye X, Jia RY, Chen W, Yu T, Zheng XD, Chu Q. Radix Tetrastigma flavonoids inhibit the migration and promote the apoptosis of A549 cells both in vitro and in vivo. J Funct Foods. 2020;72: 104076.

41. Sun Y, Li HY, Hu JN, Li J, Fan YW, Liu XR, Deng ZY. Qualitative and Quantitative Analysis of Phenolics in Tetrastigma hemsleyanum and Their Antioxidant and Antiproliferative Activities. J Agr Food Chem. 2013;61: 10507.

42. Lou T, Ji T, Peng X, Ji W, Yuan L, Wang J, Li S, Zhang S and Shi Q. Extract From Tetrastigma hemsleyanum Leaf Alleviates Pseudomonas aeruginosa Lung Infection: Network Pharmacology Analysis and Experimental Evidence. Front Pharmacol. 2021;12: 587850.

43. Sun Y, Hui QR, Chen R, Li HY, Peng H, Chen F, Deng ZY. Apoptosis in human hepatoma HepG2 cells induced by the phenolics of Tetrastigma hemsleyanum leaves and their antitumor effects in $\mathrm{H} 22$ tumor-bearing mice. J Funct Foods. 2018;40: 349.

44. Kirtia A, Prasharb V, Kumara A, Pandeya S, Rajaram H. Thymidylate kinase (TMK) of the photosynthetic, nitrogenfixing cyanobacterium Nostoc sp. strain PCC7120: Biophysical, biochemical and physiological characterization. Plant Physiol Bioch. 2021;166: 416. 
45. Colasante C, Zheng F, Kemp C, Voncken F. A plant-like mitochondrial carrier family protein facilitates mitochondrial transport of di- and tricarboxylates in Trypanosoma brucei. Mol Biochem Parasit. 2018;221: 36.

46. Pazderník M, Mareš J, Pilný J, Sobotka R. The antenna-like domain of the cyanobacterial ferrochelatase can bind chlorophyll and carotenoids in an energy-dissipative configurationBinding of pigments on the ferrochelatase $C A B$ domain. J Biolog Chem. 2019;294: 11131.

47. Yang JF, Lin M, Jiang WB, Yao Y, Tang YH, Pang YZ. Comprehensive identification and characterization of abiotic stress and hormone responsive glycosyl hydrolase family 1 genes in Medicago truncatula. Plant Physiol Bioch. 2021;158: 21.

48. Tsuge $\mathrm{Y}$, Kato N, Yamamoto S, Suda M, Inui M. Enhanced production of d-lactate from mixed sugars in Corynebacterium glutamicum by overexpression of glycolytic genes encoding phosphofructokinase and triosephosphate isomerase. J Biosci Bioeng. 2019;127: 288.

49. Jiang D, Li P, Yin Y, Guang X, Chun R, Liu S. Molecular cloning and functional characterization of UGTs from Glycyrrhiza uralensis flavonoid pathway. Int J Biol Macromol. 2021;192: 1108.

50. Shao DN, Li YJ, Sun J. GhGSTF12, a glutathione S-transferase gene, is essential for anthocyanin accumulation in cotton (Gossypium hirsutum L.). Plant Sci. 2021;305: 110827.

51. Dixon DP, Edwards R. Roles for Stress-inducible Lambda Glutathione Transferases in Flavonoid Metabolism in Plants as Identified by Ligand Fishing. J Biolog Chem. 2010;285: 36322.

52. Qian S, Qiao L, Xu W, Jiang K, Wang Y, Lin H. An inner filter effect-based near-infrared probe for the ultrasensitive detection of tetracyclines and quinolones. Talanta. 2019;194: 598.

53. Song W, Liu YX, Fanady B, Han YF, Xie L, Chen ZY, Yu K, Peng X, Zhang XL, Ge ZY. Ultra-flexible light-permeable organic solar cells for the herbal photosynthetic growth. Nano Energy. 2021;86: 106044.

54. Peng X, Li M, Wu H, Chen H, Zhang Z. Co-regulation Role of Endogenous Hormones and Transcriptomics Profiling Under Cold Stress in Tetrastigma hemsleyanum. J Plant Growth. Regul. 2021;40: 1992.

55. Peng X, Wu H, Chen H, Zhang Y, Qiu D, Zhang Z. Transcriptome profiling reveals candidate flavonol-related genes of Tetrastigma hemsleyanum under cold stress. BMC Genomics. 2019;20: 687.

56. Huson DH, Buchfink B. Fast and sensitive protein alignment using DIAMOND. Nat Methods. 2015;12: 59.

57. Langmead B, Salzberg SL. Fast gapped-read alignment with Bowtie 2. Nat Methods. 2012;9: 357.

58. Langfelder P, Horvath S. WGCNA: an R package for weighted correlation network analysis. BMC Bioinformatics. 2008;29: 559.

\section{Figures}



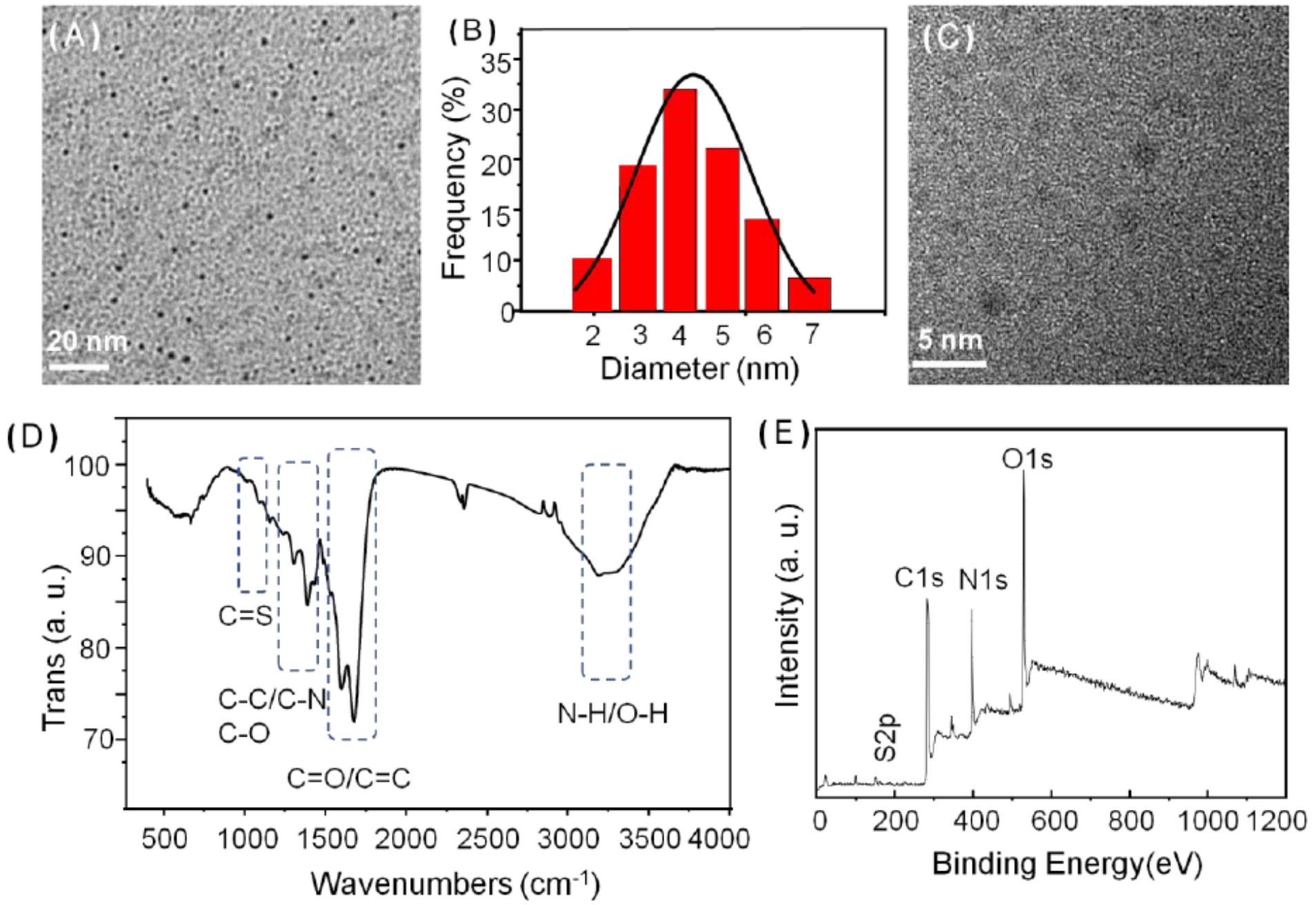

Figure 1

(A) TEM image of the NIR-CDs, and the corresponding size distribution (B). HR-TEM (C), FT-IR spectrum (D) and XPS

(E) characterizations of the obtained NIR-CDs.
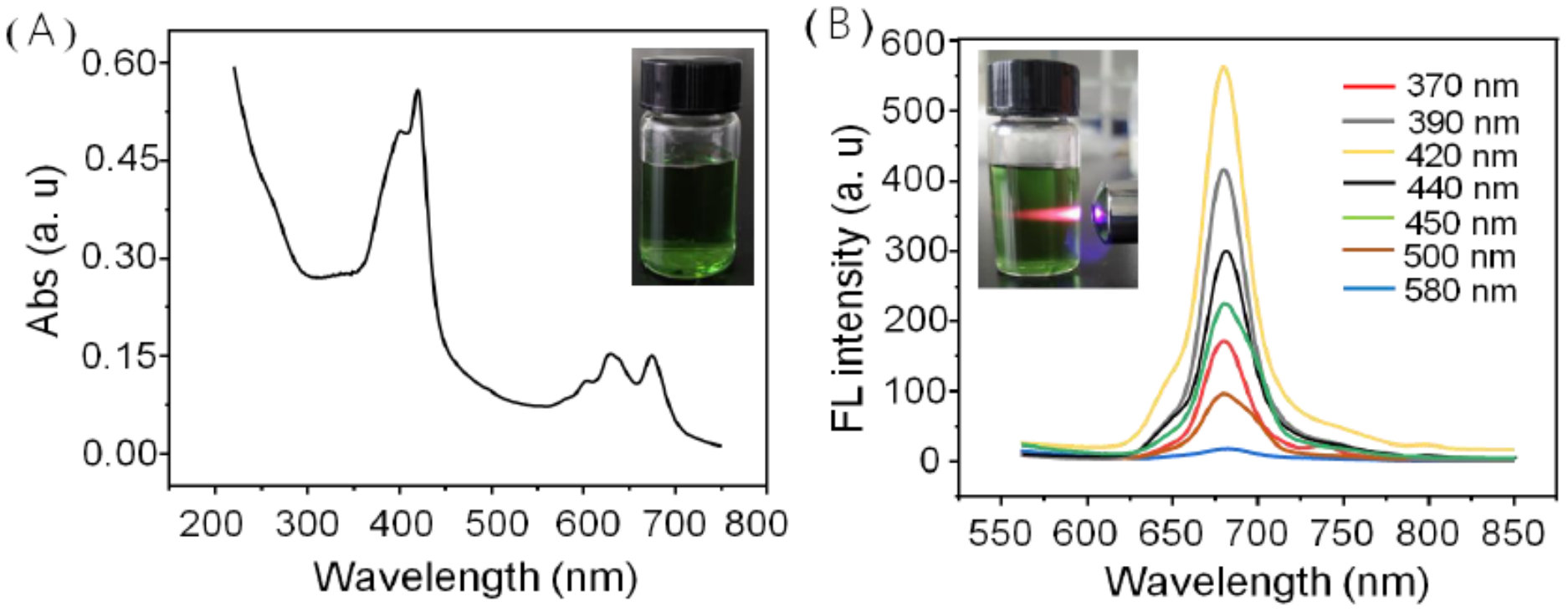

Figure 2 
(A) Uv-vis absorption spectrum of the NIR-CDs $(100 \mu \mathrm{g} / \mathrm{mL})$, inset: digital photograph of the NIR-CDs solution under daylight. (B) Fluorescence emission spectra of the NIR-CDs $(20 \mu \mathrm{g} / \mathrm{mL})$, inset: digital photograph of the NIR-CDs solution under the excitation of laser pointer (405 nm).

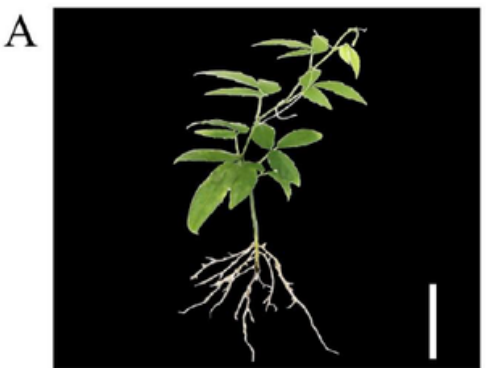

NIR-CDs

Root
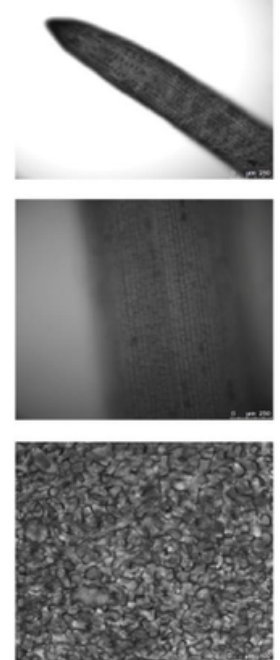

Bright field

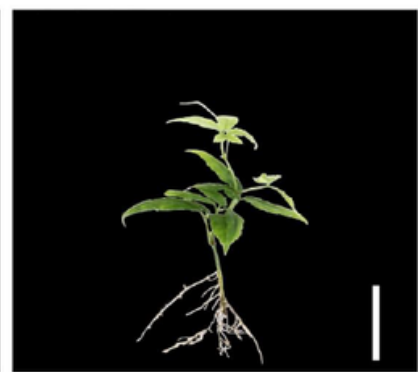

CK
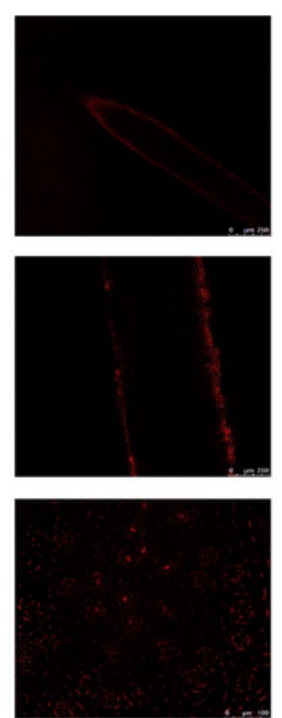

514 nm excitation
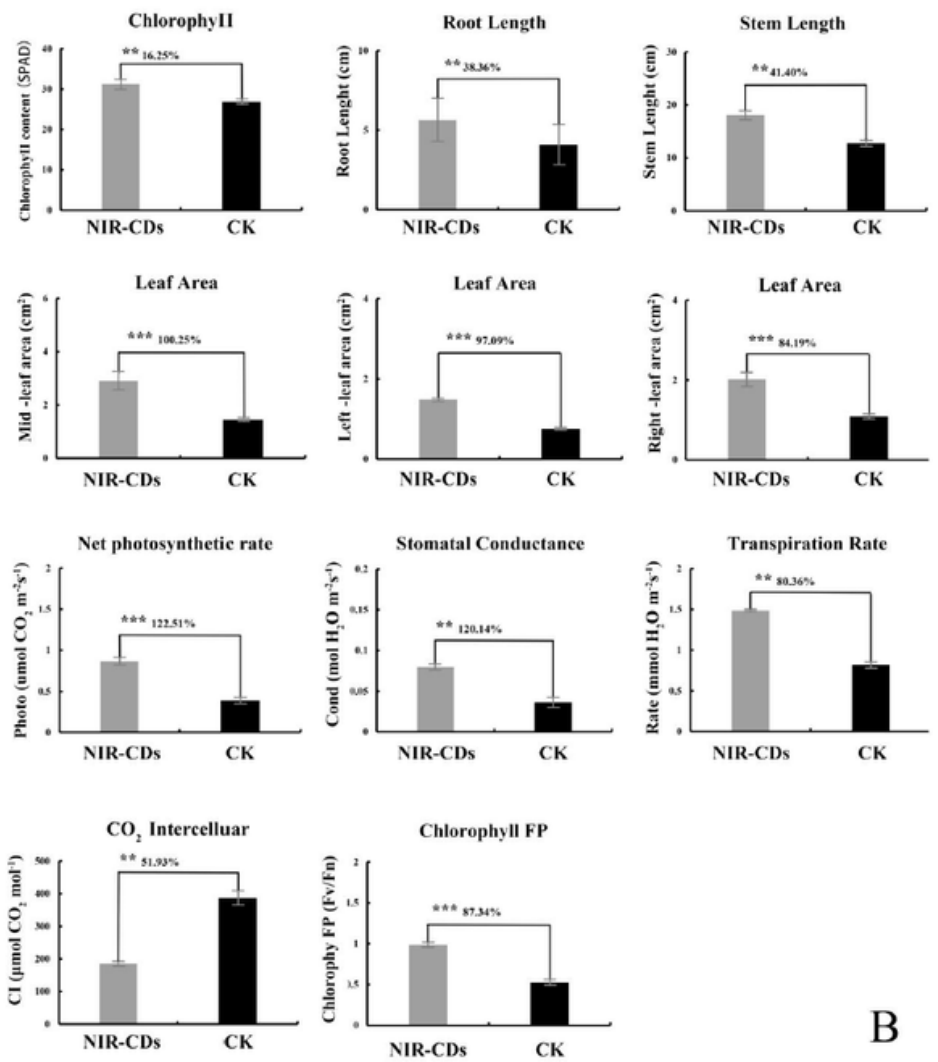

B

\section{Figure 3}

(A) The phenotypic and Laser scanning microscopy (LSM) images of root, stem, and leave cultured with NIR-CDs (0.05 $\mathrm{mg} / \mathrm{mL}$ ) (scale bar: $10 \mathrm{~cm}$ ). (B) Effect of NIR-CDs $(0.05 \mathrm{mg} / \mathrm{mL})$ on the growth potential indexes and photosynthetic parameters of T. hemsleyanum during 30 days. All the experiments were repeated three times at least. Marked with * $(P<0.05)$, ** $(P<0.01)$ and $* \star *(P<0.001)$ exhibit significant differences from control, respectively. 

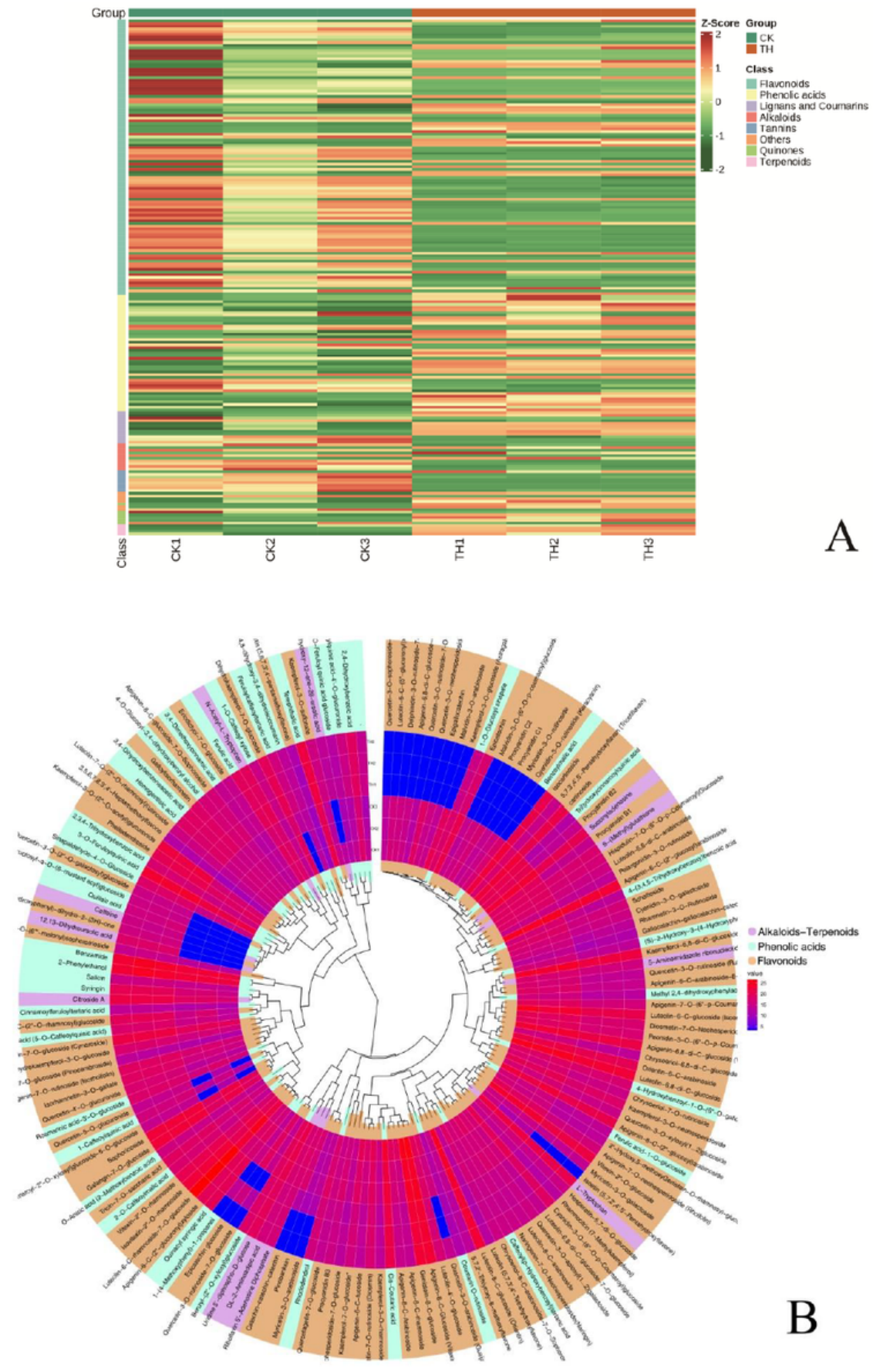

Figure 4

(A) The overall heatmap of 8 categories of secondary metabolites among CK and TH. (B) The heatmap of the fold changes of secondary metabolites among $\mathrm{CK}$ and $\mathrm{TH}$ of $\mathrm{T}$. hemsleyanum. The color bar represents the normalized fold change values. Three categories of secondary metabolites were marked, including flavonoids (claybank), phenolic acids (nattier blue), and alkaloids/terpenoids (purple). 
A

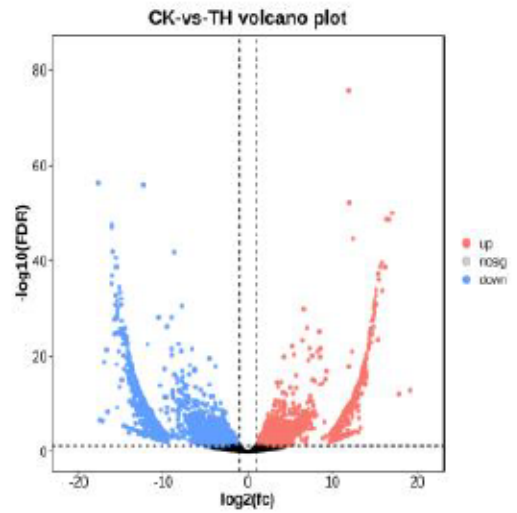

B

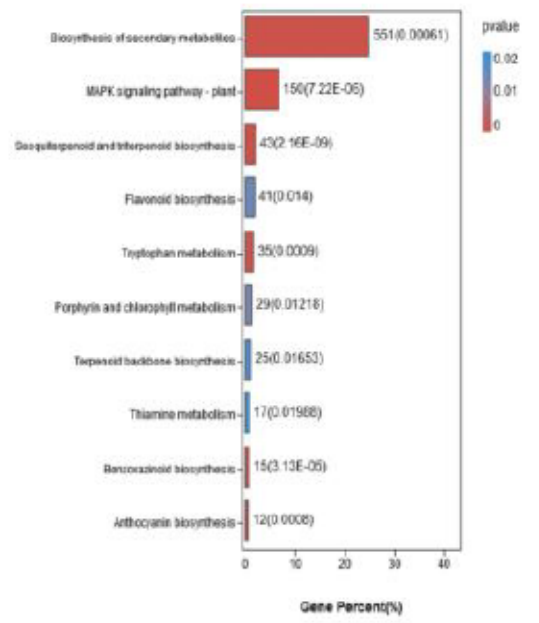

C

D

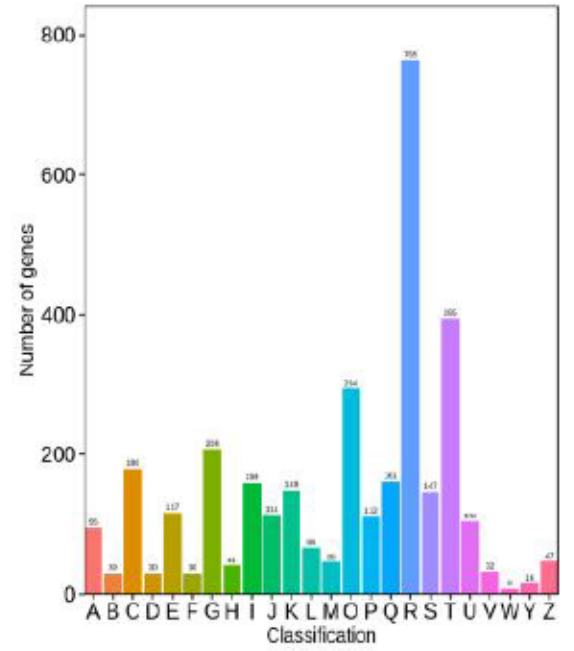

D. Cel cycte conrol cet divsion, chronoscne paritioning is: Cel wallimeribraneiervelope Hogerests

teriame

Choessing and mocilicaten

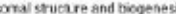

: Feclication reportination and repar

Eneray productoon and commesion

Arino acd transport and retatols

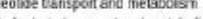

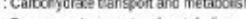

Coenzyme tansport and matakcis

P: : inongaric ion transpot ard metsedian

Q.Secunday metwo

5: Function uninomn

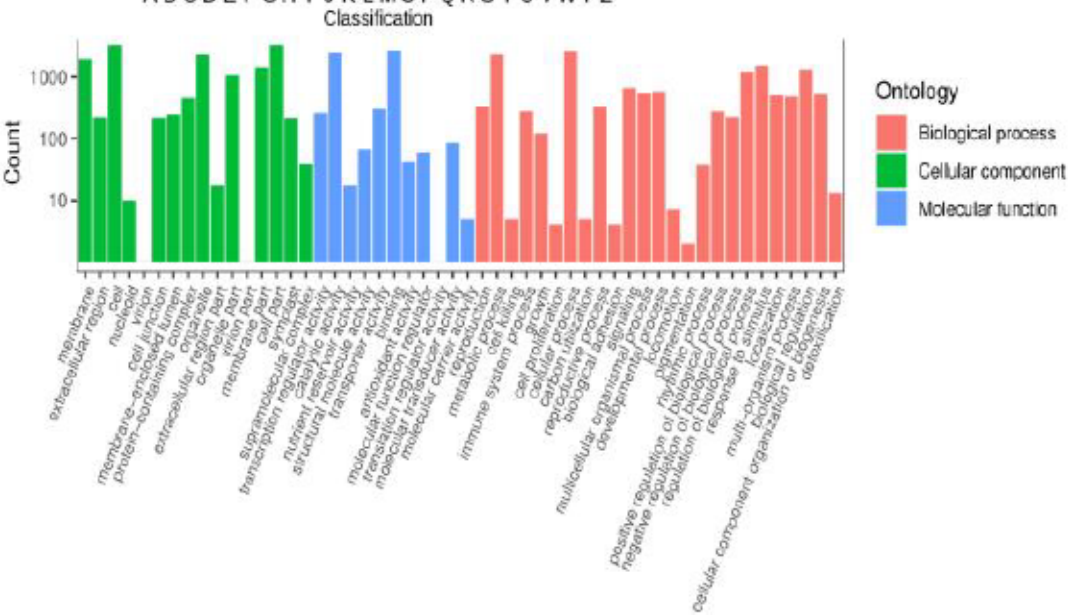

Figure 5

(A) Volcano plot of differential gene expression analysis. (B) KEGG pathway analysis for differential gene expression analysis. (C) KOG orthologous classification of differential gene. (D) GO level terms classification of differential genes. 
A

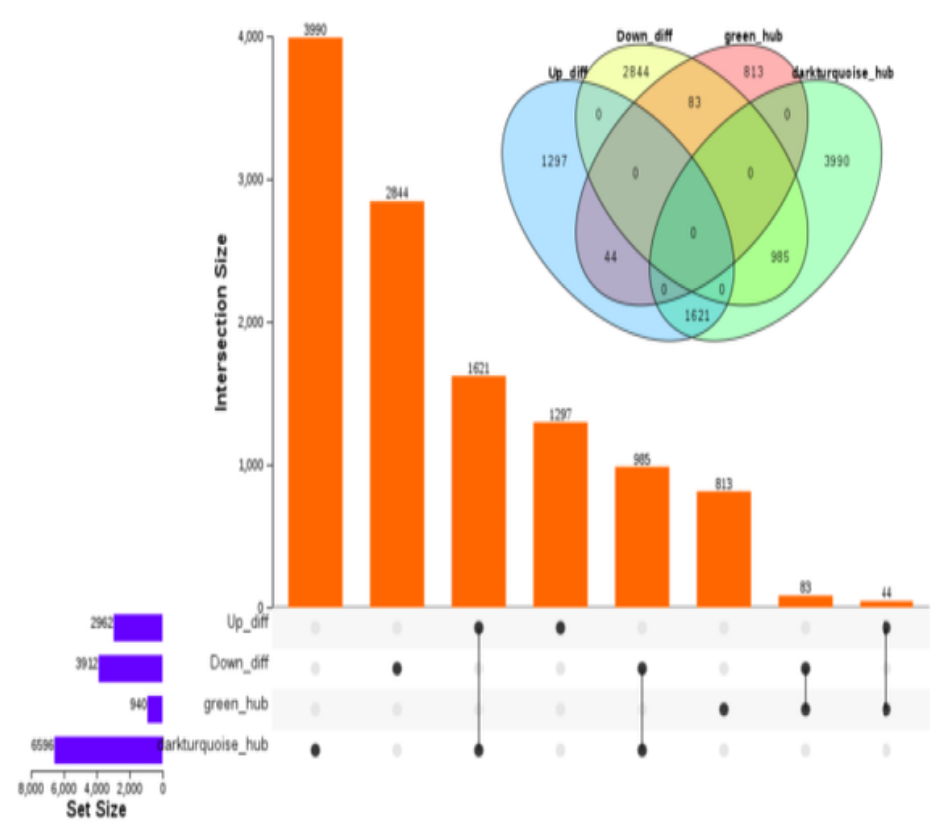

B

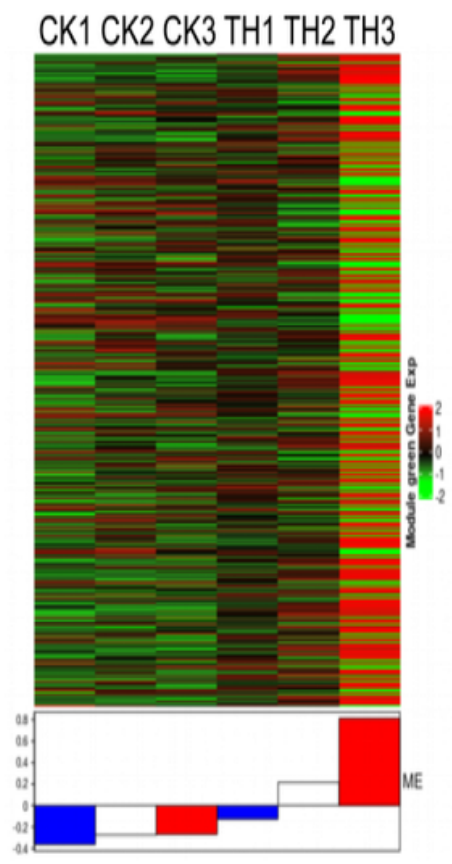

C darkturquoise

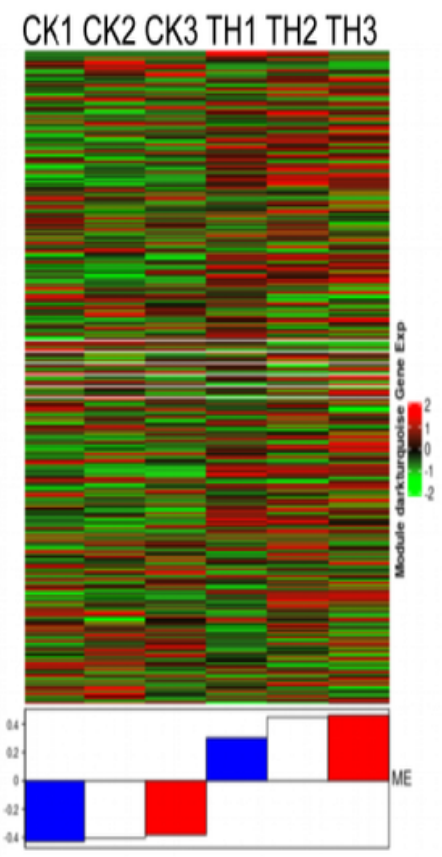

Figure 6

(A) Correlation Venn diagram of up-down regulated genes and phenotypic related gene module members. (B) Expression pattern of green module gene and column graphs of gene significance. (C) Expression pattern of darkturquoise module gene and column graphs of gene significance. 
A
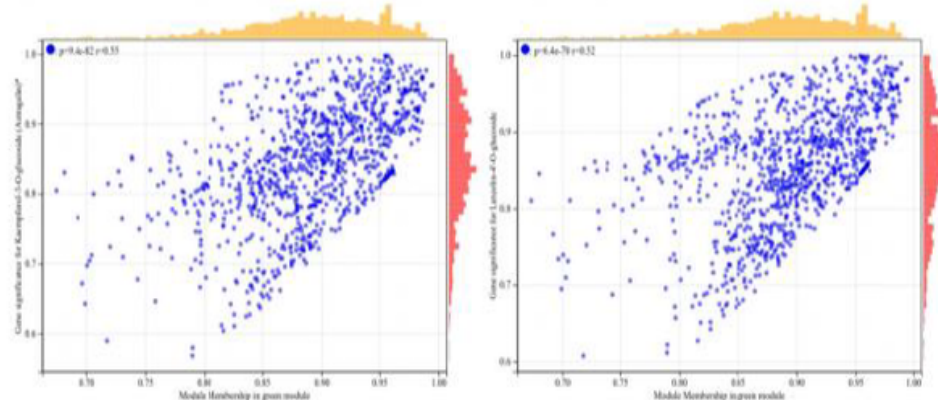

Kaempferol-3-O-glucoside (Astragalin)

Luteolin-4'-O-glucoside

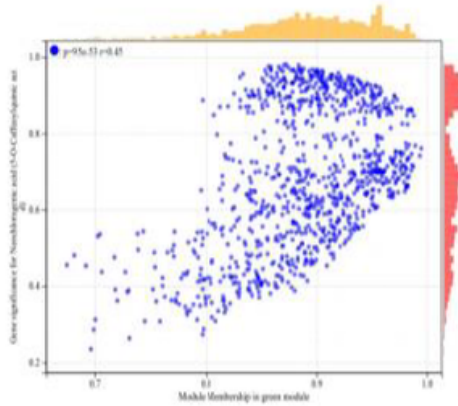

Neochlorogenic acid

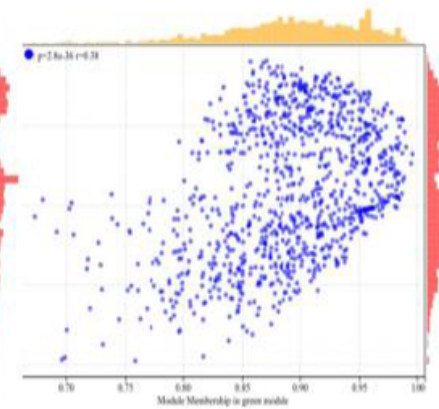

1-Caffeoylquinic acid
B

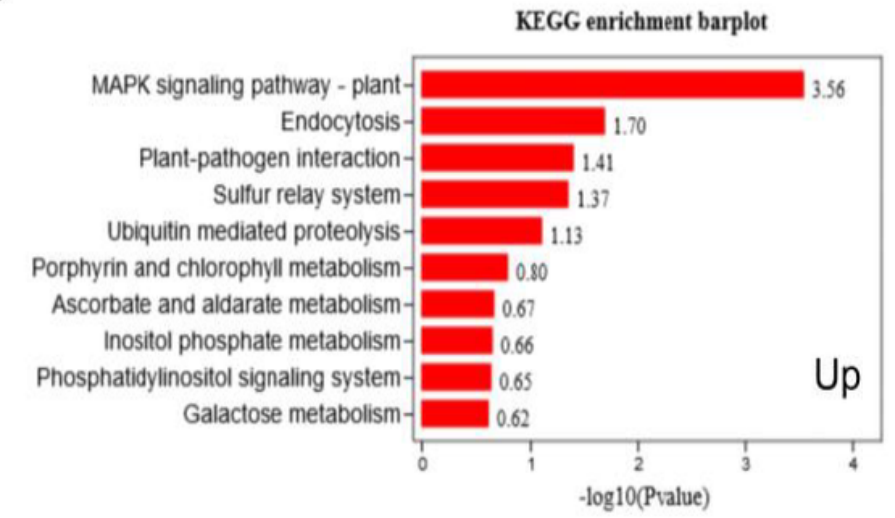

C

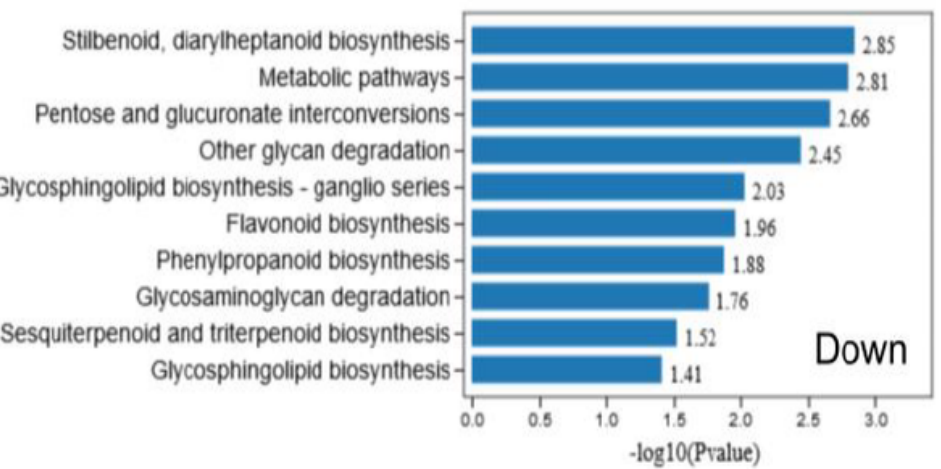

Figure 7

(A) Scatter plot of correlation between kaempferol-3-o-glucoside (astragalin), neogenetic acid (5-o-caffeoylquinic acid), 1-caffeoylquinic acid, luteolin-4'- 0-glucoside and green module. (B) The top 10 enriched pathways of up-regulated genes in the green module by KEGG. (C) The top 10 enriched pathways of down-regulated genes in the green module by KEGG. 
A
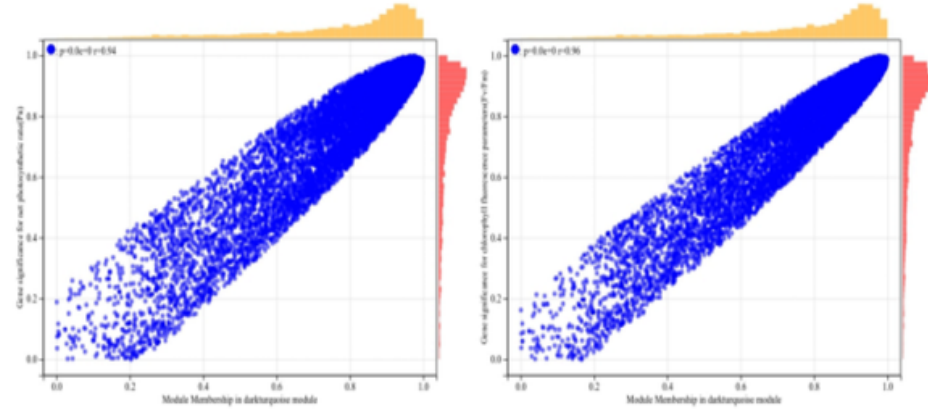

net photosynthetic rate $(\mathrm{Pn})$ chlorophyll fluorescence parameters $(\mathrm{Fv} / \mathrm{Fm})$

B

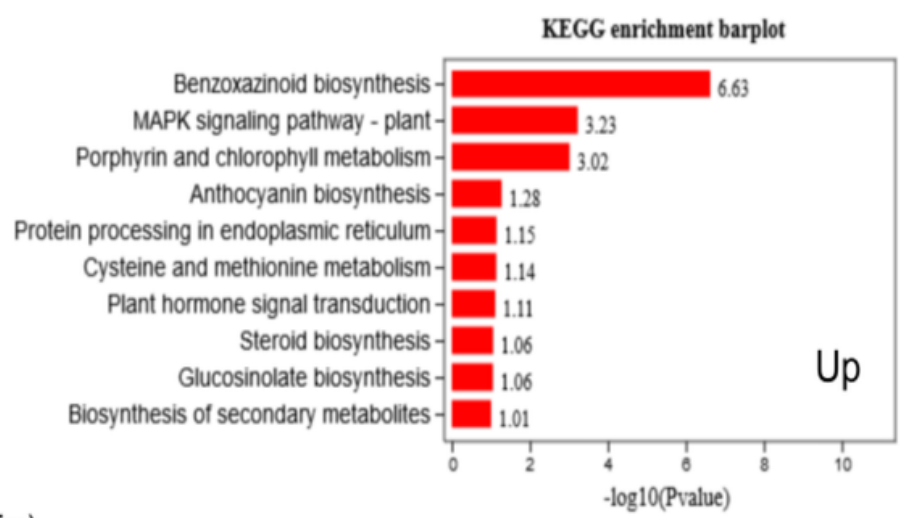

C
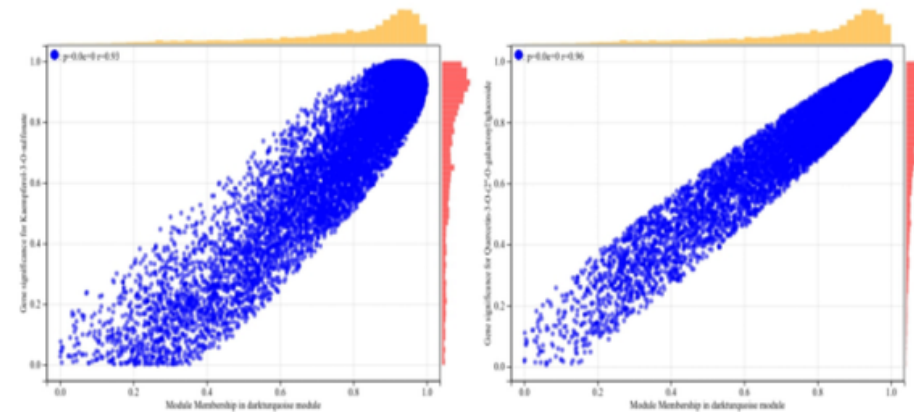

Kaempferol-3-0-sulfonate

Quercetin-3-0-(2"-0-galactosyl)glucoside

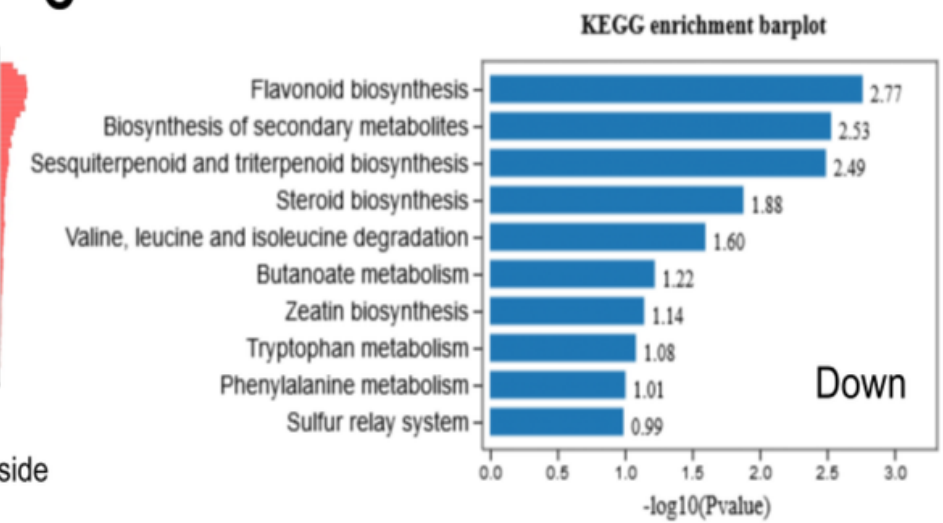

\section{Figure 8}

(A) Scatter plot of correlation between net photosynthetic rate (Pn), chlorophyll fluorescence parameters (Fv/Fm), kaempferol-3-0-sulfonate, quercetin-3-0-(2"-0-galactosyl) glucoside and darkturquoise module. (B) The top 10 enriched pathways of up-regulated genes in the darkturquoise module by KEGG. (C) The top 10 enriched pathways of downregulated genes in the darkturquoise module by KEGG. 

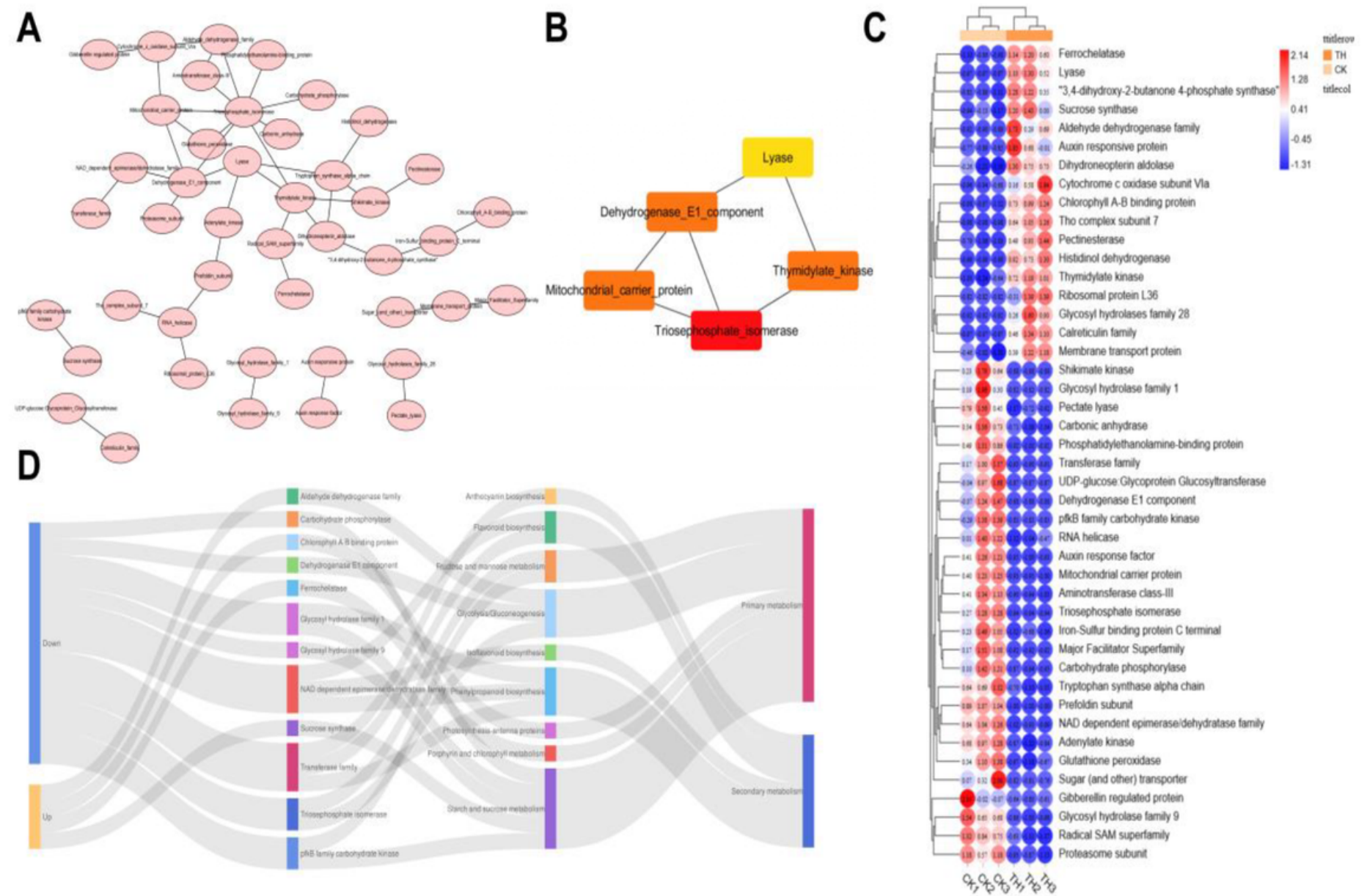

Figure 9

(A) Protein-protein interaction network. (B) The top 5 hub genes of screened the most important protein network nodes by MCC algorithm. (C) The expression pattern of node genes in PPI network. (D) The sanky plot of gene information in PPI network.

A
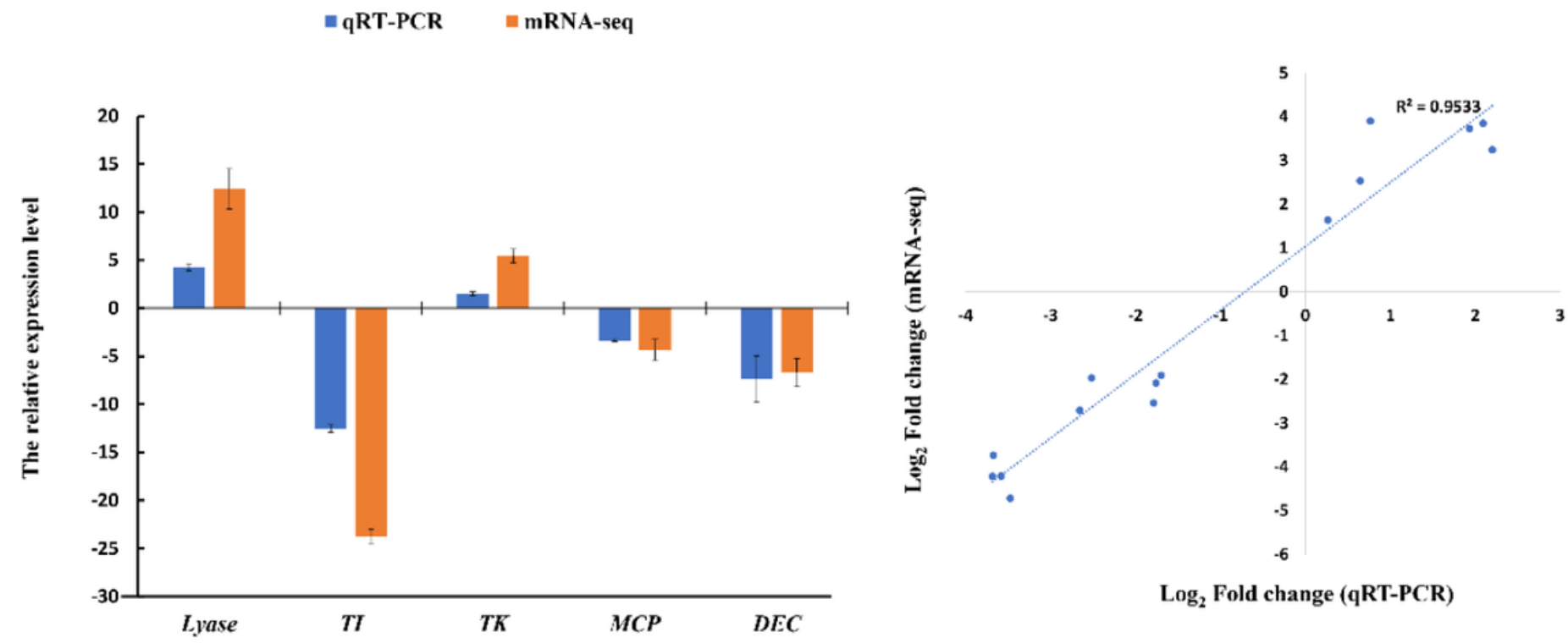

$\log _{2}$ Fold change (qRT-PCR)

Figure 10 
(A) Verification of RNA-Seq sequencing data by the qRT-PCR assay. Lyase, Triosephosphate isomerase (TI), Thymidylate kinase (TK), Mitochondrial carrier protein (MCP), Dehydrogenase E1 component (DEC). (B) Pearson correlation coefficients of the log2 of gene expression ratios obtained from RNA-seq data and qRT-PCR. All qPCR data were collected from three biological replicates and three technical replicates for each sample.

\section{Supplementary Files}

This is a list of supplementary files associated with this preprint. Click to download.

- Tables1.xIsx

- Tables2.xIsx

- TableS3.xisx

- Fig.S1.png

- Fig.S2.png

- Fig.S3.png

- Fig.S4.png

- FigureS5.pdf

- FigureS6.jpg

- FigureS7.jpg

- Figures8.pdf 